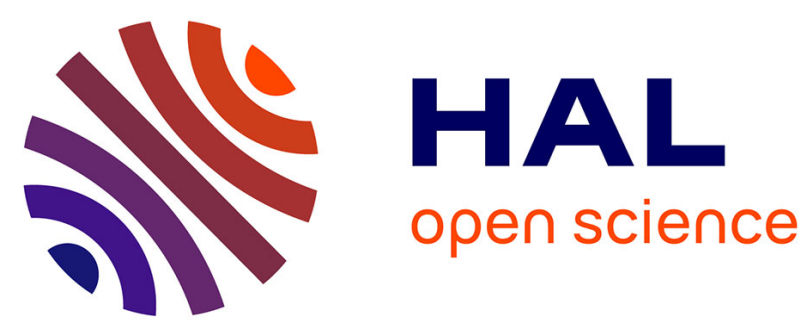

\title{
Numerical Prediction of Multiscale Electronic Conductivity of Lithium-Ion Battery Positive Electrodes
}

François Cadiou, A. Etiemble, T. Douillard, François Willot, O. Valentin, Jean-Claude Badot, Bernard Lestriez, E. Maire

\section{- To cite this version:}

François Cadiou, A. Etiemble, T. Douillard, François Willot, O. Valentin, et al.. Numerical Prediction of Multiscale Electronic Conductivity of Lithium-Ion Battery Positive Electrodes. Journal of The Electrochemical Society, 2019, 166 (8), pp.A1692-A1703. 10.1149/2.1221908jes . hal-02137415v2

HAL Id: hal-02137415

https://hal.science/hal-02137415v2

Submitted on 20 Dec 2019

HAL is a multi-disciplinary open access archive for the deposit and dissemination of scientific research documents, whether they are published or not. The documents may come from teaching and research institutions in France or abroad, or from public or private research centers.
L'archive ouverte pluridisciplinaire HAL, est destinée au dépôt et à la diffusion de documents scientifiques de niveau recherche, publiés ou non, émanant des établissements d'enseignement et de recherche français ou étrangers, des laboratoires publics ou privés. 


\title{
Numerical prediction of multiscale electronic conductivity of
}

\section{Lithium-ion battery positive electrodes}

F. Cadiou ${ }^{1}$, A. Etiemble ${ }^{2}$, T. Douillard ${ }^{1}$, F. Willot ${ }^{3}$, O. Valentin ${ }^{4}$, J-C. Badot ${ }^{5}$, B. Lestriez ${ }^{* 6}$, and E. Maire ${ }^{1}$

${ }^{1}$ MATEIS, INSA Lyon, Villeurbanne, France

${ }^{2}$ Pôle Matériaux et Structures, ECAM Lyon, Lyon, France

${ }^{3}$ Center for Mathematical Morphology, Mines Paristech, Fontainebleau, France

${ }^{4}$ Aurock, Albi, France

${ }^{5}$ Institut de Recherche de Chimie Paris, Chimie-Paristech CNRS, Paris, France

${ }^{6}$ IMN Institut des Matériaux Jean Rouxel, Universté de Nantes CNRS, Nantes, France,

Reference: Journal of The Electrochemical Society, 2019, 166 (8), pp. A1692-A1703.

DOI: 10.1149/2.1221908jes. This is a "post-print" version. A few typos corrected; units in Figs. 3, 7 and pp. 19, 21 have been amended (changes highlighted in blue). Results unchanged.

\begin{abstract}
The electronic conductivity, at the multiscale, of lithium-ion positive composite electrodes based on $\mathrm{LiNi}_{1 / 3} \mathrm{Mn}_{1 / 3} \mathrm{Co}_{1 / 3} \mathrm{O}_{2}$ and/or carbon-coated $\mathrm{LiFePO}_{4}$, carbon black and poly(vinylidene fluoride) mixture is modelled. The electrode microstructures are acquired numerically in 3D by X-ray tomography and FIB/SEM nanotomography and numerically segmented to perform electrostatic simulations using Fast Fourier Transform (FFT) method. Such simulations are easy and quick to perform because they are directly computed on the grid represented by the voxels in the 3D volumes. Numerical results are compared with experimental measurements of the multiscale electronic conductivity by broadband dielectric spectroscopy (BDS). A good prediction is realised for the bulk conductivities of the $\mathrm{C} / \mathrm{LiFePO}_{4}$ phase and the CB/PVdF mixture. The combination of X-ray and FIB/SEM tomography, FFT simulation method, and BDS is thus well adapted to understand the influence of the electrode composition and microstructure on its electronic conductivity.
\end{abstract}

\footnotetext{
*Corresponding author: Bernard.Lestriez@cnrs-imn.fr
} 


\section{Introduction}

Lithium-ion batteries (LiB) are nowadays widely used to store energy in nomad devices such as laptops and smartphones. Another field of application for LiB is the automotive industry, including electric cars, hybrid electric vehicle, plug-in hybrid electric vehicles $[1,2]$. In those cases, both high power and high energy density $\mathrm{LiB}$ are required to extend the vehicles autonomy range and to reduce the charge duration.

Electrodes for LiB are complex composite materials obtained by mixing the active material (AM) powder with an electronic conductive additive and a polymeric binder in a solvent. The electrode slurry is then coated on a metallic foil that will serve as current collector. After solvent evaporation, the electrode films are pressed and assembled with a separator to form a battery cell. The electrodes and separator porosity are impregnated by a liquid electrolyte to allow the battery cell to work. It is well established that battery performance depends significantly on the electrons and ions transport properties of the composite electrodes, which are critically affected by their nano-, meso- and macrostructure through the interfacial areas, material connectivities, and the transport length scales $[3,4,5,6,7,8]$.

In the past years, implementation of X-ray computed tomography (XRCT) and focused ion beam combined with scanning electron microscopy (FIB-SEM) techniques has enabled the quantification of the composite electrodes architecture. These novel three dimensional characterisation methods allow one to interpret transport properties and eventually assess the limiting factors in electrochemical performance $[9,10,11,12,13,14,15,16,17,18]$. The reconstructed 3D geometries, obtained with these imaging methods, can subsequently be used as inputs to model the electrochemical behaviour at the microstructure scale [19, 20, 21, 22, 23]. Combined with experiments, these methods reduce the time and cost resources needed to design new batteries, when compared to empirical approaches. The present work focuses on the modelling of electronic transport properties, which are crucial with respect to electrochemical performance $[24,25,26,27,6,28,29]$, and are often less studied than ionic mass transport properties. Indeed, it is often assumed ionic transport is a limiting factor, especially under fast charge, while electronic transport is not, due to the presence of conductive additive.

Simulation methods, on this topic, have evolved from numerically-generated microstructures (ideal particle geometries as in Chen et al. [30] or Awarke et al. [31], to actual microstructures acquired by XRCT or FIB/SEM. For computation at the particle scale, Chen et al. and Awarke et al. used packings of spherical or ellipsoidal elements to generate a finite element mesh representing the electrode microstructure. Chen et al. accounted for the presence of the mixture of carbon black and polymer binder by including a uniform coat- 
ing surrounding active material particles. In Awarke et al., the active and the conductive additive particles were randomly mixed; while the binder phase was not modelled. However, its volume was accounted for by varying the void volume fractions [32]. Both studies aimed at calculating the percolation transition. Above the percolation threshold an electrically connected continuous conductive network is formed and an abrupt change leading to a much enhanced transport behaviour occurs. For Awarke et al., percolation threshold is between 7.81 and $11.31 \%$ (volume fraction) depending on the AM particle size. According to Chen et al., the percolation is dependent on the AM volume fractions as these authors are using a uniform coating on the AM particle. As AM volume fraction is over 30\% (theoretical threshold of $29 \%$ for spheres) percolation is achieved. They also have tried ellipsoidal graphite fibers and platelets and they have found a percolation threshold ranging from $7.5 \%$ to $10 \%$ (volume fraction). Both studies gave interesting trends to be followed for optimising the effective conductivity of composite electrodes with respect to the particle size ratio or shape factor. However, they fell short in taking into account the actual distribution of the mixture of conductive additive and binder that critically depends on the electrode processing route [33,6]. Recently, Usseglio-Viretta et al. [34] have quantified the percolation of the Carbon binder additive domain for positive NMC and negative graphite electrodes, based upon a physics-based numerical generation of the additive domain, within real three-dimensional electrode geometries reconstructed from X-ray computed tomography, described in [35]. While no percolation path has been found for the carbon-binder embedded within the graphite (4.6-6.0vol\% carbon-binder), the authors found a connected cluster for the NMC electrode (10.614.6\%vol carbon-binder). When compared to Awarke et al. and Chen et al. the threshold values are are different because the latter studies used numerically generated microstructures with ideal particle shapes on the contrary to [34], and also because different AM particle sizes and shapes were considered.

Imaging techniques in 3D provide a wealth of information on the microstructure. One of the main difficulties, nevertheless, is to account for the presence of widely separated length scales. The development of XRCT allows one to obtain 3D maps of actual microstructures but only of the active material (AM) phase. This is because, with this technique, the mixture of the binder with the conductive additive particles (referred to as the conducting mixture for simplicity in the following) is difficult to separate from the porosity in terms of attenuation contrast. XRCT based simulation was used to calculate conductivity using the thermal/electric analogy and finite element method in Cooper et al. [18]. This kind of simulations provides a fairly good insight on the conductivity in the active material phase but ignores the role of the conducting mixture, which is a strong approximation as it is the main electron conducting phase. Grillet et al. [25] used XRCT as a basis to generate realistic AM microstructures and, in their simulations, assumed that the conducting mixture uniformly coated the surface of the AM particles. The authors also investigated the effect of the 
deterioration of the conduction of this conducting mixture upon ageing on the variation of the electrode effective conductivity upon cycling. However, such uniform coating assumption may not be representative of the actual mixture spatial distribution, as it may form some aggregates of various size and shape more or less randomly spatially distributed as well as some dendritic, fractal-like, structures constituting a 3D connected (or not) network through the electrode thickness. For instance, different carbon-binder mixture morphology, ranging from filmlike to tentaclelike, can be generated through a physics-based process related with interfacial energy [35]. Kashkooli et al. [20] tried to account for this morphological characteristic of the conducting mixture, which however was not considered separately from the active material phase, but rather as random bridges between neighbouring active particles. An electrical conductivity was accordingly postulated for the obtained reconstructed microstructure and used to simulate the electrochemical (charge and discharge) behaviour. Wieser et al. [36] used the higher resolution of FIB/SEM to image in 3D the conducting mixture. The authors studied the contribution of this nanoporous phase in the mass transport restriction of the electrolyte salt species within the electrode. Inoue et al. [37] studied realistic $\mathrm{LiCoO}_{2}$ and graphite electrode microstructures obtained from FIB/SEM reconstructions and evaluated their electronic conductivities. The electronic conductivity was calculated assuming that the electron transport through the electrode could be modelled with homogenisation theory. This simply allowed them to calculate the effective electronic conductivity of a composite from the bulk conductivity in the different phases, their volume fractions and the tortuosity of the transport path, which were all obtained from the FIB/SEM reconstructions.

The electronic conductivity of composite electrodes for lithium batteries has also been predicted using full-field numerical computations with finite element method [38, 39] or else [40]. Contrarily to effective medium theories, this approach allows one to estimate the fields at the local scale in the microstructure while taking into account the "exact" morphology of the electrode. In the present paper, electrode microstructures designed for electric vehicle application, including $\mathrm{LiNi}_{1 / 3} \mathrm{Mn}_{1 / 3} \mathrm{Co}_{1 / 3} \mathrm{O}_{2}$ (NMC) and/or carbon coated $\mathrm{LiFePO}_{4}$ (LFP) as active material, carbon black (CB) as electronic conductive additive and poly(vinylidene fluoride) (PVdF/CB) as binder, were acquired by XRCT and FIB/SEM, and numerically segmented to perform electrostatic simulations using Fast Fourier Transform (FFT) methods. Simulations are computed directly on the regular grid represented by the voxels in the 3D volumes without meshing. This approach was originally developed for mechanics [41] and extended to conductivity [42, 43]. This method is applied here for the first time to Lithium-ion battery composite electrodes. The simulation results are compared to experimental measurements of the electronic conductivity done on the same electrodes or on the constituents taken separately. This allows us to establish relationships between the electronic conductivity of the different phases in the composite electrode, its actual architecture and its effective electronic conductivity. 


\section{Digitalisation of the electrode microstructure}

The electrodes considered in the present article have been studied in previous works. Two electrodes denoted $\mathrm{F}$ and $\mathrm{H}$ are based on a blend of LFP and NMC, while a third one, denoted J, is solely based on LFP. The names $\mathrm{F}, \mathrm{H}$ and $\mathrm{J}$ are the same as those used in $[3,12]$. The electrode volumes were acquired using the porous non-impregnated electrodes with XRCT and FIB $/$ SEM techniques at a voxel resolution of $320 \times 320 \times 320 \mathrm{~nm}^{3}$ and $10 \times 10 \times 10 \mathrm{~nm}^{3}$ respectively, as described in [12].

$\mathrm{XRCT}$ volumes were acquired in phase contrast mode in order to be able to separate the NMC clusters from the other components in the electrodes. At this scale $(\sim$ scale 1$)$, the electrode thickness is entirely included in the field of view. A simple segmentation process, based on grey level thresholding, allows us to obtain segmented volumes ( $c f$. Figure 3 ) and thus the NMC volume fractions in these volumes (Table 1). X-Ray tomography volumes were segmented using a combination of simple grey level thresholdings. First the NMC particles were extracted by thresolding. Then another thresholding allowed us to extract the homogenised phase. The combination of these two thresholds enables the volume reconstructions. Internal porosity (along with some cracks created by the sample preparation method) is the complementary part of these two solid phases. No artificial intermediate layer has been noticed through volume inspection. In order to get segmented volumes without cracks, we used the same first threshold to obtain NMC particles. The complementary being the homogenised phase with internal NMC porosity. To distinguish both features, the internal NMC porosities are detected with a "fill holes" type algortihm (Fiji software) on the NMC phase and the difference with the non filled phase. This gives a good result only because the NMC clusters are quite well separated, thus only internal porosities are closed and not possible closed spaces between NMC clusters. This last part was verified by careful eye inspection of the NMC-filled volumes.

For FIB/SEM volumes, after acquisition, the image processing was performed with a home-made Fiji macro [12] which is based on the algorithm developed in [44] for the shine through artefact correction of FIB/SEM images. Briefly, the shine through artefacts stem from the fact that SEM imaging retrieves data from inside the pores. This creates a gradient, by adding data from matter below the current observation plane, that is perturbing grey levels commonly used for segmentation. This is discussed and explained further in $[12,44]$. As explained in [12], the Fiji macro is used to distinguish the solid phase from porosity. Then, active materials (NMC and LFP) and PVdF/CB mixture were clearly identified by thresholding the grey levels of BSE data. This methodology is illustrated in the Supporting Information. FIB-SEM reconstructions 
of electrodes $\mathrm{F}, \mathrm{H}$ and $\mathrm{J}$ are shown in Figure 1 together with an example of an X-Ray volume reconstruction for sample F. The raw data are available upon request to the authors for researchers eventually interested.
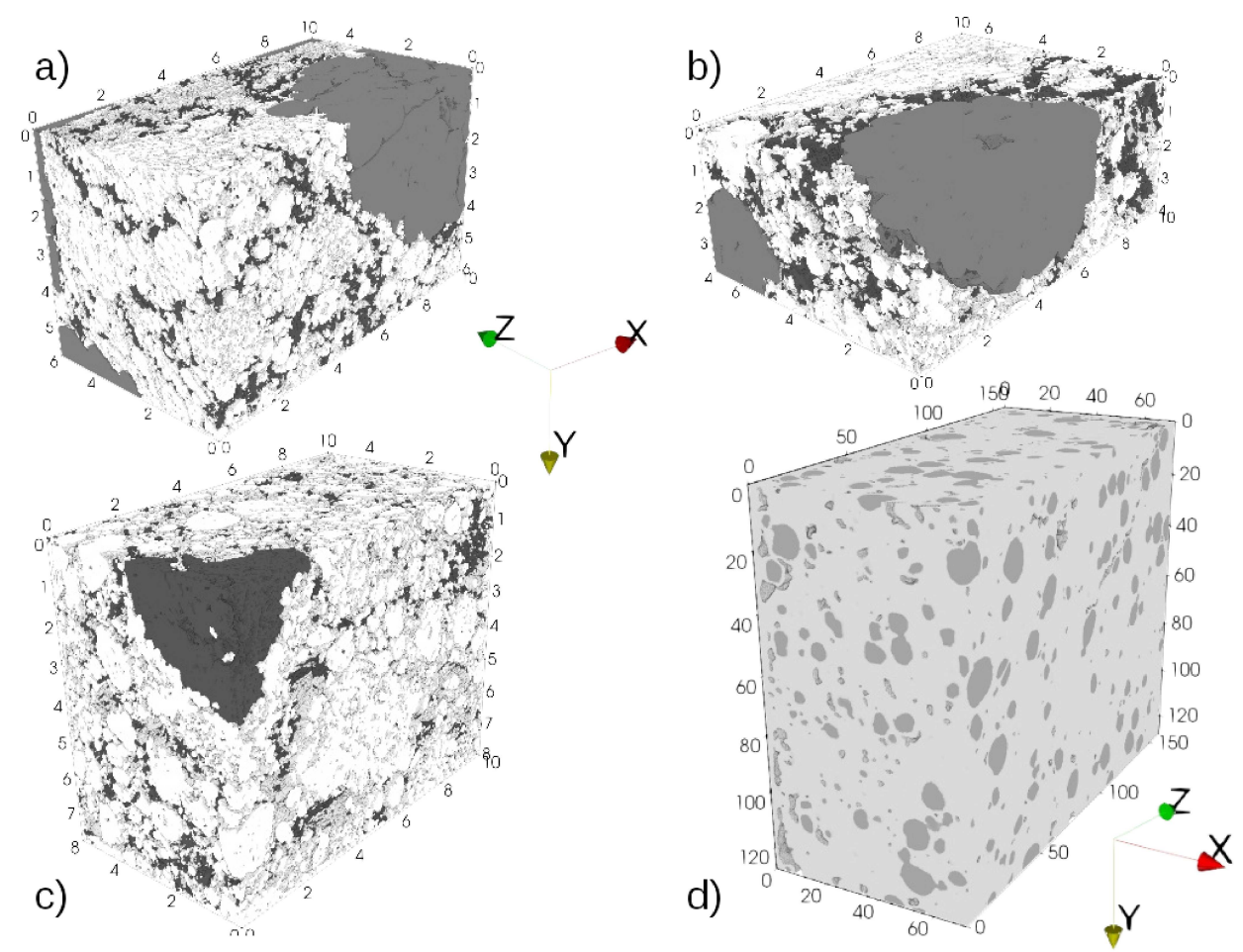

Figure 1: 3D entire segmented volumes acquired by FIB/SEM a), b) and c) and X-Ray tomography (d) and used in simulations, a) Sample H, b) and d) Sample F, c) Sample J. For FIB/SEM samples, white is LFP, light grey is NMC, dark grey is the CB/PVdF mixture and the porosity is transparent for all three volumes. For X-Ray samples light grey is also the NMC clusters and the off-white/lighter grey colour is the homogenised phase (LFP, CB/PVdF, and porosity). Dimensions reported on the different axes are in $\mu \mathrm{m}$.

LFP (in white) presents the shape of nanometric particles, from few tens of nanometres to around 800nm, with some of them aggregated as clusters of particles of a few micrometers. $\mathrm{LiFePO}_{4}$ particles are covered by a nanometre thin layer of amorphous carbon, which is invisible at the FIB/SEM resolution. The carbon content is about 2wt\% in the LFP active material. NMC (in light grey) is in the form of large quasi-spherical clusters $(\sim 10 \mu \mathrm{m})$ of smaller grains. Particle size distribution for NMC and LFP phases composing the electrodes studied here have been previously reported in [12]. The PVdF and CB appear as one indiscernible mixture (in dark grey). CB is a fine graphite powder with high degree of crystallinity. The elementary particles are spheres made up of the stacking of broken, quasi-graphitic, layers. These elementary particles (mean diameter $\sim 40 \mathrm{~nm}$ ) are fused together by chemical bounds in various forms of aggregations, called primary aggregates with average diameter of 100-300nm [45]. Attractive interactions between CB and the PVdF in the electrolyte slurry favour their coagulation [46, 47]. Thus, in composite electrodes PVdF and CB are generally intimately mixed and associated into one indiscernible PVdF/CB conducting phase [48]. We believe that, with our $10 \mathrm{~nm}$ voxel size, we can access pores of size above $20 \mathrm{~nm}$. Therefore, the smaller pores 
within the PVdF/CB mixture might be invisible for us. Vierrath et al. [49] used FIB-SEM tomography to reconstruct the carbon-binder domain of a $\mathrm{LiCoO}_{2}$ electrode with contrast enhancement by $\mathrm{ZnO}$ infiltration via atomic layer deposition. They found the porosity inside the carbon domain is about $58 \%$ with the poresize distribution centred at $64 \mathrm{~nm}$. According to their results, we should have access to around $75 \%$ of the pores within the PVdF-CB domains. The last phase present in the studied samples is a mostly open porosity (intraconnectivity above $96 \%$ in all cases), which is filled with a liquid electrolyte in functional conditions to conduct the lithium ions.

Table 1 gives the volume fraction $\phi_{v}(\%)$ of the LFP, NMC, PVdF/CB and porosity phases, and intraconnectivity of the LFP and PVdF/CB phases, at various electrode scales. These different scales are depicted in Figure 2. Scale 0 is the entire macroscopic electrode. The values reported in the table for this scale are average values measured macroscopically from the electrode thicknesses, areas, weights and the material densities. Scale 1 is the XRCT volume and scale 2 is the FIB/SEM volume for which the values were measured in [12] using standard 3D image analysis. Scale 3 represents a small subdomain within the FIB/SEM volume. The peculiarity of this sub-volume is that it does not contain the large NMC particles. Such subdomains will be used in the multiscale simulations discussed in this work. Simulation results are shown in section 5. Starting from the experimentally measured electronic conductivity for each electrodes, the effective conductivity of the $\mathrm{LFP} / \mathrm{PVdF} / \mathrm{CB} /$ porosity homogenised phase will be determined with the XRCT volumes. Then this conductivity will be considered as the effective one for the FIB/SEM volumes, and the conductivity of the LFP and the PVdF/CB phases will be then determined. These ones will be compared to reference values.

In Table 1, we quantify the intraconnectivity as the volume fraction of the most voluminous cluster of one material/phase in the analysed volume. A value of $100 \%$ of this parameter means that all voxels of this material/phase are part of a same unique percolated object in the analysed volume. $\mathrm{F}$ and $\mathrm{H}$ are made with NMC/LFP (50:50 mass ratio) as active material and J only contains LFP. The PVdF/CB phase volume fractions in electrodes $\mathrm{F}, \mathrm{H}$ and $\mathrm{J}$ are respectively 16.4, 11 and 9.1\%. The $\mathrm{PVdF} / \mathrm{CB}$ phase is quasi fully percolated within the FIB/SEM volumes of $\mathrm{F}$ and $\mathrm{H}$ (intraconnectivity at scale 3 equals to $97 \%$ and $96 \%$ respectively), while it is rather not in $\mathrm{J}$ (intraconnectivity at scale 3 equals to $22 \%$ ). The LFP phase fully percolates within the FIB/SEM volumes. One can notice some differences between volume fractions measured from tomographic data and standard measurements (scale 0). These differences can come from a representativity issue (as will be discussed after) and have also been addressed in [12] from a manufacturing point of view. 
The tortuosities given in Table 1 are geometric ones and these are measured along the y axis of Figure 1 (through the electrode thickness) to comply with the direction of the imposed potential difference used in the simulation developed in Sections 4 and 5. By definition, the geometric tortuosity is the length of the shortest path between two locations, going only through the phase of interest, divided by the straight distance between these two locations.

$$
\tau=\frac{\text { shortest path length }}{\text { straight distance }}
$$

It is then always superior to 1 . In this work, the tortuosity is calculated by the following procedure. A seed plane is initially defined in the middle of the binarised tomographic volume, perpendicular to the $\mathrm{y}$ axis. Then, a front is propagated ( pixel agglomeration through dilation) only in the phase of interest, materialising the length of the tortuous path. Then, at the end of the propagation procedure, each reachable voxel in the phase of interest is labelled with its distance from the seed plane. A measurement of the average distance from the seed plane can then easily be calculated in each plane perpendicular to the seed plane. The so-calculated distance is however not equal to the true euclidean distance and depends on the criterion used to propagate the front. A criterion based on six neighbours (the voxel is added if it shares a common face with one of the front voxels) overestimates the distance while a criterion based on 26 neighbours (the voxel is agglomerated if it shares a common face, edge, or summit with one of the front voxels) underestimates the distance. In the present study, we make the calculation twice (with 6 and 26 neighbours). Then, with respects to the straight distance from the seed plane, a linear interpolation allows us to calculate a mean tortuosity in the phase (one for 6 neighbours and one for 26 neighbours). And, as mentioned, we estimate the geometrical tortuosity as the average of both measurements. 
Table 1: Properties of the volumes used for the simulation. $\phi_{v}$ stands for volume fraction. Source 0 corresponds to (real) macroscopic properties from the manufacturer. Other sources refer to the imaging techniques used to acquire the volume. Corresponding dimensions in $\mu \mathrm{m}$ are reported in the Size column which has been formatted like $\mathrm{A} \times \mathrm{B} \times \mathrm{C}$ with $\mathrm{C}$ the dimension along the electrode thickness

\begin{tabular}{|c|c|c|c|c|c|c|c|c|}
\hline \multirow[t]{2}{*}{ Sample } & \multirow[t]{2}{*}{ Source } & \multirow[t]{2}{*}{ Size $\left(\mu \mathrm{m}^{3}\right) *$} & \multicolumn{4}{|c|}{ Volume fraction $\phi_{v}(\%)$} & \multicolumn{2}{|c|}{$\begin{array}{c}\text { Intraconnectivity (\%) / } \\
\text { Geometric tortuosity }\end{array}$} \\
\hline & & & NMC & LFP & $\mathrm{PVdF} / \mathrm{CB}$ & Pores & LFP & $\mathrm{PVdF} / \mathrm{CB}$ \\
\hline \multirow{4}{*}{$\mathrm{F}$} & 0 & - & 24 & 30.6 & 16.4 & 29 & - & - \\
\hline & $\mathrm{XRCT}$ & $128 \times 160 \times 72$ & 18.5 & \multicolumn{3}{|c|}{81.5} & - & - \\
\hline & FIB/SEM & $10 \times 4 \times 7$ & 20.4 & 36.1 & 10.0 & 33.5 & $99.8 / 1.17$ & $87 / 1.25$ \\
\hline & FIB/SEM & $2.3 \times 2.3 \times 2.3$ & - & 37.8 & 18.5 & 43.7 & $98.5 / 1.36$ & $96 / 1.35$ \\
\hline \multirow{4}{*}{$\mathrm{H}$} & $\mathbf{0}$ & - & 25.5 & 32.5 & 11 & 31 & - & - \\
\hline & $\mathrm{XRCT}$ & $128 \times 160 \times 67.8$ & 21.1 & \multicolumn{3}{|c|}{78.9} & - & - \\
\hline & FIB/SEM & $10 \times 6 \times 5.4$ & 15.4 & 49.4 & 10.6 & 24.6 & $99.7 / 1.11$ & $90.8 / 1.39$ \\
\hline & FIB/SEM & $4 \times 4 \times 4$ & - & 54.3 & 16.3 & 37 & $99.6 / 1.1$ & $95 / 1.34$ \\
\hline \multirow{3}{*}{$\mathrm{J}$} & $\mathbf{0}$ & - & - & 53.9 & 9.1 & 37.0 & - & - \\
\hline & FIB/SEM & $10 \times 8 \times 5.5$ & - & 58.5 & 8.1 & 33.4 & $99.8 / 1.09$ & $23 /-$ \\
\hline & FIB/SEM & $5 \times 5 \times 5$ & - & 64.9 & 4.7 & 30.4 & $99.9 / 1.08$ & $17.6 /-$ \\
\hline
\end{tabular}

* F, $\mathrm{H}$ and $\mathrm{J}$ thickness are respectively $77.5,72$ and $91.5 \mu \mathrm{m}$ (including the current collector).

One can notice that no more NMC enter the composition of $\mathrm{F}$ and $\mathrm{H}$ volumes for the last source from FIB/SEM. This is explained in Figure 2 and serves the purpose of multiscale simulation discussed in this work.

The sign - means that the property is not measurable. 


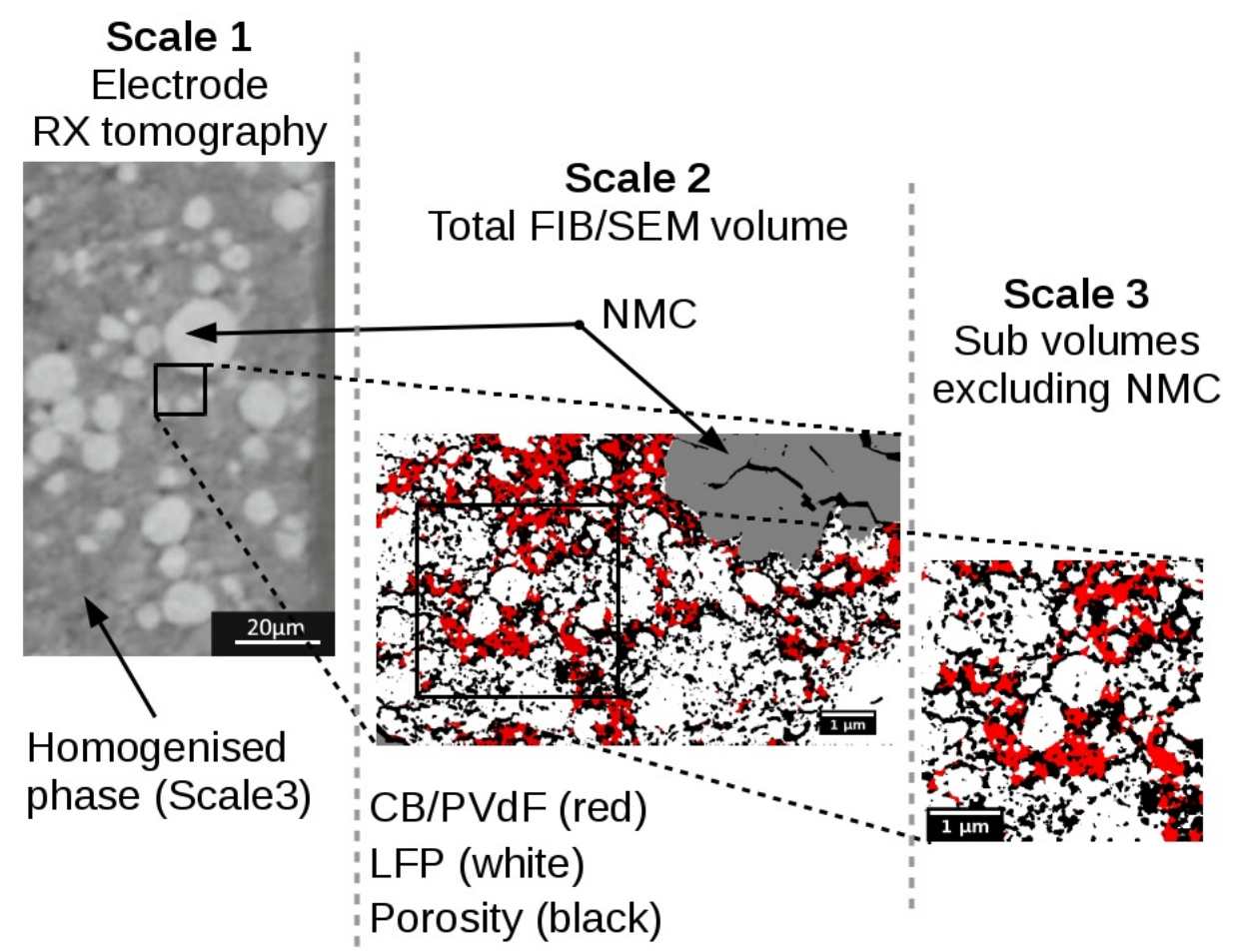

Figure 2: Multiscale view for the microstructures studied. The homogenised phase in the X-Ray tomography picture is composed of the $\mathrm{PVdF} / \mathrm{CB}$, LFP and porosity phases.

\section{Electrical conductivity measurements}

The electronic conduction properties were acquired with Broadband dielectric spectroscopy (BDS). The theoretical background and experimental setup to implement this technique are well described in previous papers. See for example Badot et al. [50]. With the BDS technique, it is possible to measure the electrical properties at different scales (sample, cluster, particle and atom) of an electrode material. When a time-dependent electric field is applied to the material, polarisations (charge-density fluctuations) are produced. The timescale (or relaxation time) of the polarisation depends on the scale at which it occurs and on the conductivity at this scale. In electronic conductors, different relaxation mechanisms generally result from their crystal structure, nanostructure and microstructure. Because they have distinct characteristic frequencies, they can be separated and treated individually. All details about the BDS measurements are given in the Supporting Information as most of them are already published elsewhere. Results for the conductivity measurements are thus summarised in Table 2 and discussed hereafter. The interested reader is encouraged to refer to section 5.4 for comparisons of conductivity values with analytical estimates. 
Table 2: Room temperature conductivity at different scales for NMC and LFP powders (pressed pellets), $\mathrm{PVdF} / \mathrm{CB}$ mixture, electrodes F, H and J.

\begin{tabular}{|c|c|c|c|c|c|c|}
\hline \multirow{2}{*}{ Material } & \multicolumn{3}{|c|}{ Volume fractions (\%) } & \multirow{2}{*}{\multicolumn{2}{|c|}{ Conductivity $\left(\mathrm{S} \mathrm{m}^{-1}\right)$}} & \multirow{2}{*}{ Reference } \\
\hline & NMC & LFP & $\mathrm{PVdF} / \mathrm{CB}$ & & & \\
\hline \multirow{4}{*}{$\begin{array}{l}\text { NMC pellet } \\
(7 \% \mathrm{PVdF})\end{array}$} & \multirow{4}{*}{74} & \multirow{4}{*}{-} & \multirow{4}{*}{-} & Sample & 0.000035 & \multirow{4}{*}[53]{} \\
\hline & & & & Cluster & $\begin{array}{c}0.0001 \\
\text { (effective) }\end{array}$ & \\
\hline & & & & $\begin{array}{c}\text { Grain in a } \\
\text { cluster }\end{array}$ & $\begin{array}{c}0.2 \\
\text { (effective) }\end{array}$ & \\
\hline & & & & Interatomic & $\begin{array}{c}19 \\
\text { (effective) }\end{array}$ & \\
\hline \multirow{3}{*}{$\begin{array}{l}\text { LFP pellet } \\
(2 \% \mathrm{PVdF})\end{array}$} & \multirow{3}{*}{-} & \multirow{3}{*}{65} & \multirow{3}{*}{-} & Sample & 0.26 & \multirow{3}{*}{ This work } \\
\hline & & & & Coating & $\begin{array}{c}0.4 \\
\text { (effective) }\end{array}$ & \\
\hline & & & & $\begin{array}{l}\text { Intra } \mathrm{sp}^{2} \\
\text { domains }\end{array}$ & $\begin{array}{c}\quad>20 \\
\text { (effective) }\end{array}$ & \\
\hline $\begin{array}{c}\mathrm{PVdF} / \mathrm{CB} \\
\text { films }\end{array}$ & - & - & 100 & Sample & 50 to 400 & [55] \\
\hline \multirow{2}{*}{ Electrode F } & & & & Sample & 4 & \multirow{2}{*}{ [3] and this work } \\
\hline & 24 & 30.6 & 16.4 & $\begin{array}{l}\mathrm{PVdF} / \mathrm{CB} \\
\text { clusters }\end{array}$ & $\begin{array}{c}9.1 \\
\text { (effective) }\end{array}$ & \\
\hline \multirow{2}{*}{ Electrode $\mathrm{H}$} & & & & Sample & 2.94 & \multirow{2}{*}{ [3] and this work } \\
\hline & 25.5 & 32.5 & 11 & $\begin{array}{c}\mathrm{PVdF} / \mathrm{CB} \\
\text { clusters }\end{array}$ & $\begin{array}{c}4.26 \\
\text { (effective) }\end{array}$ & \\
\hline \multirow{2}{*}{ Electrode J } & & & & Sample & 0.3 & \multirow{2}{*}{ [3] and this work } \\
\hline & - & 53.9 & 9.1 & $\begin{array}{l}\mathrm{PVdF} / \mathrm{CB} \\
\text { clusters }\end{array}$ & $\begin{array}{c}4.1 \\
\text { (effective) }\end{array}$ & \\
\hline
\end{tabular}

The bare $\mathrm{LiFePO}_{4}$ compound has very low intrinsic conductivity. The conductivity of the carbon coated compound is an effect of the carbon coating in which $\mathrm{LiFePO}_{4}$ particles are embedded. It contains short-order $\mathrm{sp}^{2}$-coordinated carbon domains (graphite nanocrystallites) bonded by $\mathrm{sp}^{3}$-coordinated carbon atoms. At 
the microscopic (particle) scale, the electrical conductivity is due to electrons hopping between $\mathrm{sp}^{2}$ domains within the carbon coating around the $\mathrm{LiFePO}_{4}$ particle. At the macroscopic (sample) scale, the electrical conductivity within this phase depends on the contact resistance between the LFP particles and on the void volume fraction [51]. The conductivity at the different scales of the LFP phase is given in Table 2. It is very important to note that the measured values are effective. True conductivity could only be measured for dense, non-porous samples. For example, the conductivity of graphite is about $10^{5} \mathrm{~S}^{\mathrm{m}} \mathrm{m}^{-1}$ [52]. The effective conductivity measured for graphite nanocrystallites in the carbon coating of C-LFP is measured to be in the 20-100S. $\mathrm{m}^{-1}$ range, because the carbon volume fraction in the sample is very low, about $3.8 \%$ in C-LFP and $2.5 \%$ in the porous pellet.

The conductivity of the NMC compound stems from the presence of $\mathrm{Ni}^{3+}$ ions, which are balanced by negatively charged $\mathrm{M}$ vacancies. This enables a hole $2 \mathrm{D}$ conduction in the nickel layer. The conductivity within the NMC clusters is however limited due to blockage of the charge carriers (holes) at the interstitial grain-boundary amorphous region between NMC crystallites (grains). Furthermore, at the macroscopic (sample) scale, the electrical conductivity is hindered due to a constriction resistance at inter-clusters contacts [53].

The conductivity of the PVdF/CB phase is provided by the carbon black nanoparticles. In this mixture, CB aggregates are generally not in direct contact but are separated by an interfacial PVdF layer. The combination of percolation theory and interparticle tunnelling conduction accounts well for the electrical behaviour of such polymer/CB mixture [54, 26]. At the microscopic scale (a few aggregates), the electrical conductivity is due to the tunnelling of electrons through the thin polymer layers adsorbed between the CB aggregates. This tunnel resistivity increases exponentially with the polymer gap thickness. At the macroscopic (sample) scale, an electrical conductivity is only observed if the CB aggregates percolates through the PVdF matrix. This condition is fulfilled for the PVdF to CB mass ratios (5:1 to 1:1) typically used in composite electrodes [48]. The typical conductivity of PVdF/CB mixture was measured in [55] on dense PVdF/CB films. The conductivity values range from 50 to $400 \mathrm{~S} \cdot \mathrm{m}^{-1}$ for PVdF to CB ratios from 5:1 to 5:4.

The conductivity of the three electrodes studied here was measured by BDS and some values were already reported in [3]. These values however were here refined. Resistivity Nyquist plots for the three electrodes are given and interpreted in the Supporting Information. By having a second look at these data, it was possible to measure three parameters: (i) the contact resistance at the electrode/aluminium current collector, $\mathrm{R}_{C}$, (ii) the electrode layer conductivity, here named sample conductivity, $\sigma_{S}$, and (iii) the effective conductivity of the $\mathrm{PVdF} / \mathrm{CB}$ clusters at high frequency (in the 1-100 MHz range), $\sigma_{C}$. The sample conductivity of J is close 
to that of a pressed LFP pellet, 0.3 and $0.26 \mathrm{~S} . \mathrm{m}^{-1}$, respectively. These two conductivities show Arrheniuslike temperature dependence with similar activation energy, 0.07 and $0.09 \mathrm{eV}$. These comparisons strongly suggest that the electrode layer conductivity of $\mathrm{J}$ is dominated by the contribution of the LFP phase, this one being percolated contrarily to the PVdF/CB mixture. The sample conductivity of $\mathrm{J}$ is however higher and its activation energy is lower than that of a pure LFP pellet as J contains isolated PVdF/CB clusters, which temperature independent effective conductivity could be determined to be equal to $4.1 \mathrm{~S} . \mathrm{m}^{-1}$ as explained in the Supporting Information (Figure S2). The temperature independence of this high frequency conductivity, which is typical of the tunnelling mechanism, allows its unambiguous attribution to the $\mathrm{PVdF} / \mathrm{CB}$ unpercolated clusters.

The sample conductivity of $\mathrm{F}$ and $\mathrm{H}$ is one order of magnitude higher than that of $\mathrm{J}$. According to FIB/SEM reconstructions, these high conductivities can be explained by the percolation of the PVdF/CB mixture that likely short-circuits the less conductive LFP and NMC particles in the electrode. The effective conductivity of the $\mathrm{PVdF} / \mathrm{CB}$ clusters is higher in $\mathrm{F}$ than in $\mathrm{H}$, while it is similar in $\mathrm{H}$ and $\mathrm{J}$, depending on their different volume fractions.

\section{Modelling and computer simulation}

The simulations described here use the FFT method by solving the electrostatic equations with the Morphhom software, making use of the "discrete" Green operator introduced in [43]. This software has already been used to perform simulations on the electrical conductivity of fuel cells [40] and for the homogenisation of mechanical properties [56,57] or optical response [58]. The interest of this method is that simulations are computed directly on the regular grid represented by the voxels in the $3 \mathrm{D}$ reconstructed FIB/SEM volumes. The electrostatic equations solved here are:

$$
\operatorname{div}(J(\vec{x}))=0, E(\vec{x})=-\overrightarrow{\operatorname{grad}(\phi)}, J(\vec{x})=\sigma E(\vec{x})
$$

Where $\phi$ is the electric potential, E the electric field, J the current field and $\sigma$ is the local conductivity at point $\vec{x}$. A strong hypothesis is that all contacts are supposed to be perfect, i.e. no contact resistance nor constriction resistance between the different phases are considered. Phase continuity is assumed to occur when two voxels pertaining to the same phase are touching by their faces. The impact of the microstructure is then essentially reflected in the phase volume fraction, intraconnectivity (or extent of percolation) and 
tortuosity.

\section{Results and discussion}

\subsection{Simulations based on X-Ray tomography volumes}

In this subsection, we focus on the first simulation scale performed on X-Ray tomography volumes.

One can observe in Figure 3a) and f) that some cracks are present in the X-Ray volumes. These cracks are most certainly due to the sample preparation for tomography. In order to be imaged at such a small resolution, the sample has to be very thin (around 0.8 or $1 \mathrm{~mm}$ thick). Thus, cutting from the mother electrode is very likely to induce cracks in these fragile samples. The X-Ray samples, for $\mathrm{H}$ and $\mathrm{F}$, were then segmented in two different ways, with and without cracks, to observe their impact on the current density field. The discernible internal voids inside the NMC clusters are considered as porosity in both cases. Their volume fraction is however negligible with less than $0.01 \%$. Internal voids plus cracks together count for up to $2.9 \%$ volume fraction in the volumes. The segmentation results are shown in Figure $3 \mathrm{~b}), \mathrm{d}), \mathrm{g}$ ) and i). In these images, the white phase indicates the NMC clusters and the black one the porosities. The light grey areas symbolises an homogenised phase composed of LFP, PVdF/CB mixture and porosity at a smaller scale accessible with the FIB/SEM resolution. 

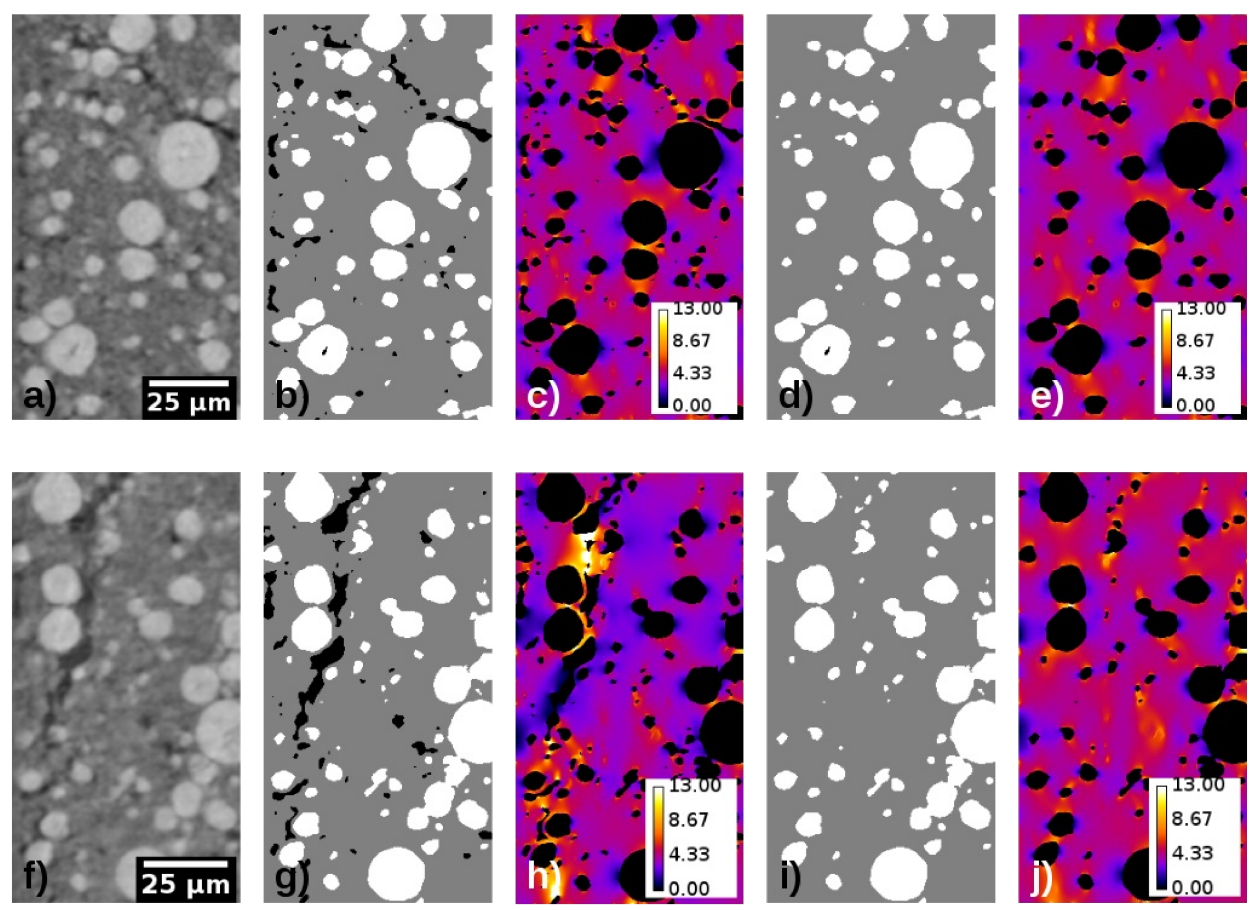

Figure 3: From a) to e) is sample $\mathrm{F}$ and from $\mathrm{f}$ ) to $\mathrm{j}$ ) is sample $\mathrm{H}$. a) and $\mathrm{f}$ ) are X-Ray original data. b) and g) segmentation with cracks and d) i) segmentation without cracks. c), h) and e), j) are respectively 2D maps of the current density field with and without cracks. These maps are sections from the 3D FFT data results. A $1 V / m$ [instead of $1 V$ ] difference potential is imposed between the left and right faces (thickness direction of the electrode sample), colour scale is expressed in A.m ${ }^{-2}$ [instead of A.m ${ }^{-1}$ ]. In the segmented images, white is for NMC, black for porosity and the light grey for an homogenised phase (LFP, PVdF/CB and porosity at the FIB/SEM smaller scale).

The FFT simulations were then carried out on both types of segmented volumes for $\mathrm{H}$ and $\mathrm{F}$. The simulations were performed by fixing the conductivity of the low conducting NMC phase to $0.0001 \mathrm{~S} . \mathrm{m}^{-1}$, according to experimental data [53] (see Table 2 and 3), and varying the conductivity of the homogenised phase, $\sigma_{v a r}$ (bulk conductivity), from $2 \mathrm{~S} \cdot \mathrm{m}^{-1}$ to $8 \mathrm{~S} \cdot \mathrm{m}^{-1}$. The effective volume conductivity, predicted by the FFT method, varies linearly with respect to $\sigma_{v a r}$. We accordingly determine for each sample the conductivity of the homogenised phase which allows one to recover the experimentally measured effective conductivity. This homogenised phase conductivity will then be used to determine the conductivities of the individualised LFP and PVdF/CB phases, by change of scale, using the FIB/SEM volumes (cf. Section 5.2). The simulation results and input parameters are gathered in Table 3. Figure 4 shows the variation of the calculated volume conductivity as a function of the homogenised phase conductivity $\sigma_{v a r}$. The horizontal lines correspond to experimentally measured sample conductivities. The homogenised phase conductivity is higher for $\mathrm{F}$ than for $\mathrm{H}$, in agreement with the higher effective conductivity in F. As expected, when cracks are considered, a higher conductivity is obtained for the homogenised phase for both electrodes. This is mostly a consequence of the more pronounced tortuosity and constrictivity of the homogenised phase, as illustrated in Figure 3. 
Table 3: Simulation results and input/target parameters for the FFT simulations on XRCT volumes

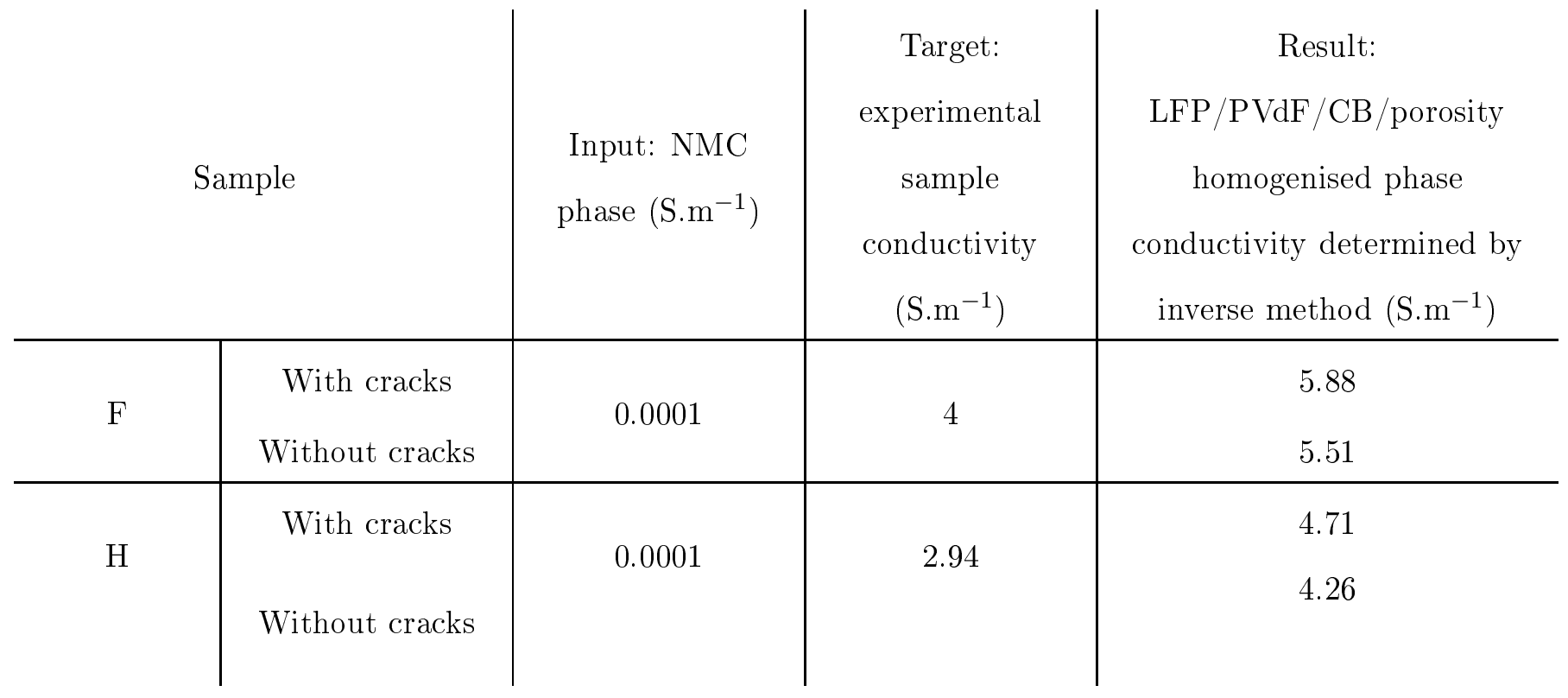

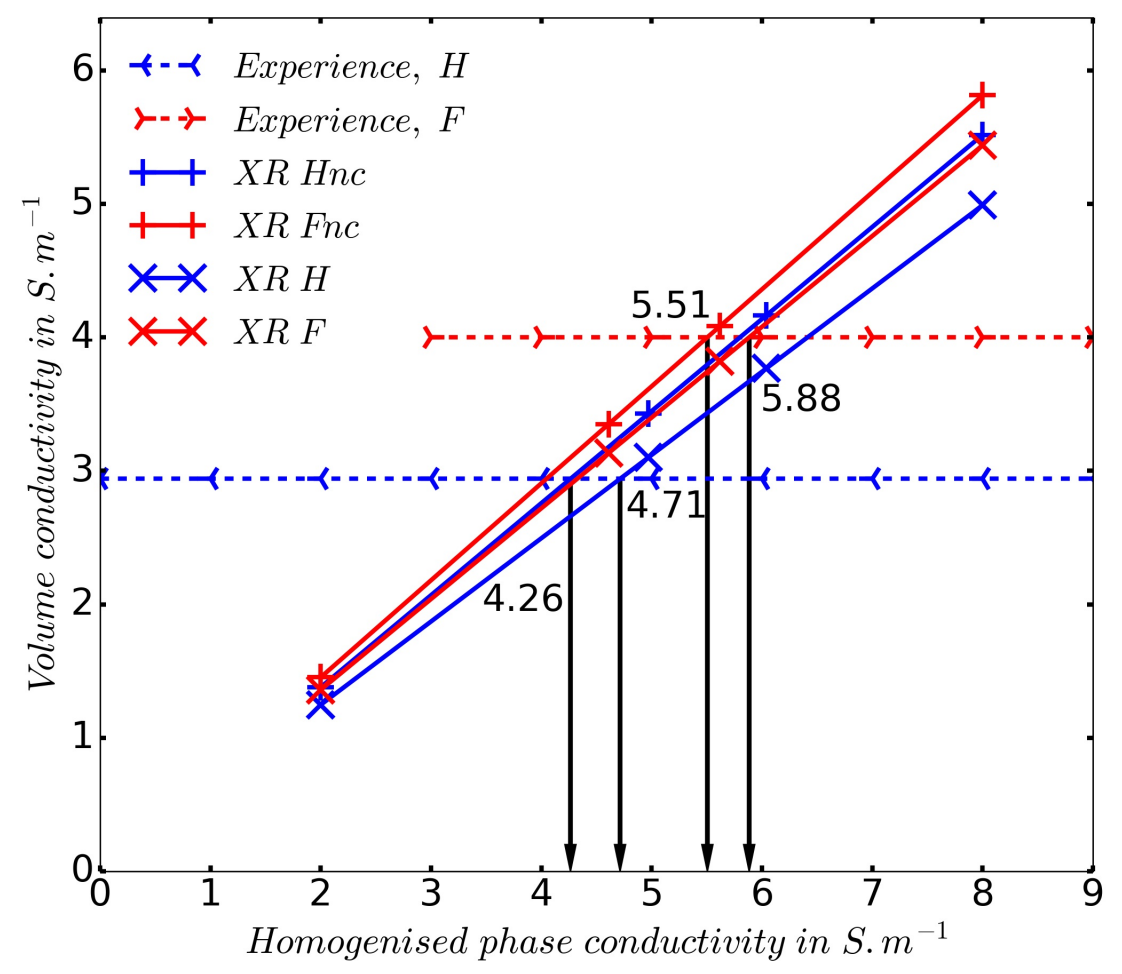

Figure 4: Effective volume conductivity of the XR volumes for samples $\mathrm{F}$ and $\mathrm{H}$ as a function of the homogenised phase conductivity. Dashed lines represent the experimental results. The text "nc" refers to volumes without cracks, otherwise the plot concerns volumes with cracks. Arrows indicate the values of the homogenized phase corresponding to experimental measurements.

The field maps of the local current density are shown in Figure 3c), e), h) and j). These fields are repre- 
sented along a 2D section. The current is enhanced in regions where the conducting phase is constricted (at bottlenecks between NMC clusters) or in cracked zones. The cracks strongly deviate the current density flow leading to highly concentrated and depleted regions, as illustrated in Figure 3h). The field fluctuations are significantly smaller in the absence of cracks (see Figure 3j). In the same way, the presence of more NMC clusters $(21.06 \%$ instead of $18.54 \%$ ) seems to also have a constriction effect in $\mathrm{H}$ compared with $\mathrm{F}$ when examining the slopes in Figure 4.

\subsection{FFT simulations on the FIB/SEM sub-volumes}

Results presented hereafter concern the sub-volumes acquired by FIB/SEM (scale 3) which are exempt of cracks contrarily to the XRCT volumes. The simulation results and input parameters are gathered in Table 4. Sample $\mathrm{J}$ is made of the homogenised phase only, within which the PVdF/CB mixture has not percolated. This makes it the best case to compute the LFP phase conductivity. It is not not possible to do so from samples $\mathrm{F}$ and $\mathrm{H}$ because of the PVdF/CB phase percolation that hinder the effect of the LFP phase conductivity. Volume fractions of the different constituents are recalled in Table 1. FFT computations are performed fixing the conductivity of the CB/PVdF mixture to $250 \mathrm{~S}^{-1} \mathrm{~m}^{-1}$, following [55] (see also Table 2), and varying the conductivity of the LFP phase $\sigma_{L F P}$. Figure 5 illustrates the variation of the sub-volume conductivity (J, $5 \mu \mathrm{m}$ edge size) as we vary the LFP phase conductivity. This is also done for two other fixed values of the $\mathrm{CB} / \mathrm{PVdF}$ phase conductivity. The effective volume conductivity varies linearly with $\sigma_{L F P}$ in the range of interest (between 0.1 and $0.6 \mathrm{~S} . \mathrm{m}^{-1}$, see Figure 5). Experimental data for the sample conductivity, i.e. $0.3 \mathrm{~S} \cdot \mathrm{m}^{-1}$ (dashed line in Figure 5), is recovered for one particular value for $\sigma_{L F P}$. We determine a LFP phase conductivity $\sigma_{L F P}$ of $0.38 \mathrm{~S} . \mathrm{m}^{-1}$ using the conductivity value of $250 \mathrm{~S} . \mathrm{m}^{-1}$ for the $\mathrm{PVdF} / \mathrm{CB}$ mixture. This value seems reasonable since it is in the same order of magnitude than the effective conductivity of the carbon coating that was measured by BDS $\left(0.40 \mathrm{~S} . \mathrm{m}^{-1}\right.$, Table 2$)$. The conductivity of the LFP phase is indeed due to the carbon coating. 


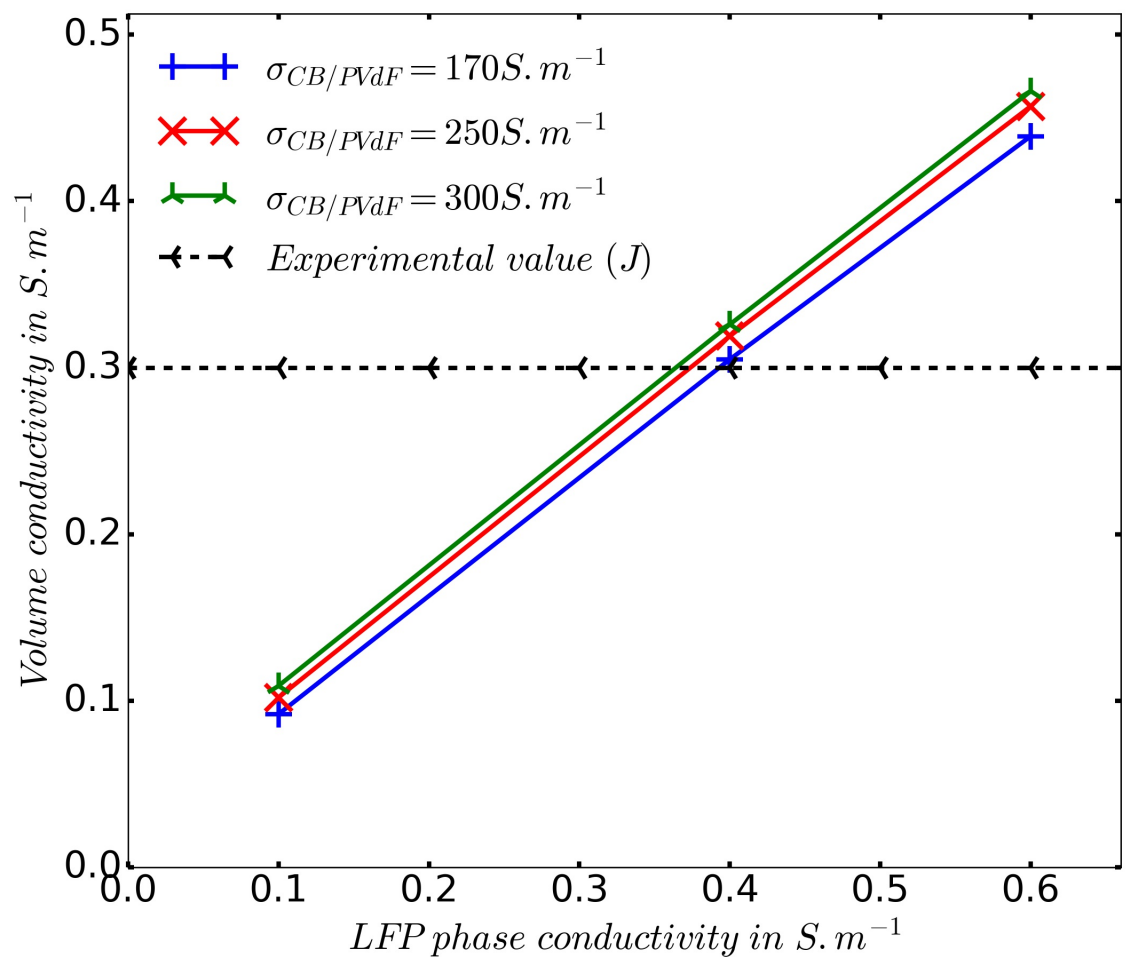

Figure 5: Variation of the effective volume conductivity for $\mathrm{J} \times 500$ as a function of the LFP phase conductivity for three fixed values of the CB/PVdF phase conductivity $\left(170,250\right.$ and $300 \mathrm{~S}^{-\mathrm{m}^{-1}}$ ) along with the experimental sample conductivity value to fit (horizontal dashed line).

In all FFT computations hereafter, we assume that $\sigma_{L F P}$ is $0.38 \mathrm{~S} . \mathrm{m}^{-1}$. We let the $\mathrm{CB} / \mathrm{PVdF}$ phase conductivity vary and compute the resulting effective conductivity for samples $\mathrm{F}$ and $\mathrm{H}$ at scale 3 . As observed previously, this results in quasi linear evolutions (see Figure 6). One can notice that these two evolutions are quite similar despite the differences in architecture and composition of the two electrodes. This can be explained by the fact that the volume fraction of the highly conductive phase (PVdF/CB mixture) is quite similar at this scale (18.5 and 16.3 for $\mathrm{F}$ and $\mathrm{H}$ respectively). The geometrical tortuosities are also really close, 1.34 for $\mathrm{F}$ and 1.35 for $\mathrm{H}$. In terms of effective volume conductivity, the slightly higher PVdF/CB volume fraction in $\mathrm{F}$ is finally possibly compensated by the much higher LFP volume fraction in $\mathrm{H}$ and/or lower porosity in $\mathrm{H}$. The horizontal dashed lines correspond to the homogenised phase conductivity values calculated using X-Ray volumes for $\mathrm{F}$ and $\mathrm{H}$ ( $c f$. Section 5.1). The computed sub-volume conductivity matches the homogenised phase conductivity of electrode $\mathrm{F}$ and $\mathrm{H}$ for a $\mathrm{PVdF} / \mathrm{CB}$ phase conductivity equal to $305 \mathrm{~S} . \mathrm{m}^{-1}$ and $210 \mathrm{~S} . \mathrm{m}^{-1}$ respectively. This gives a mean value of $258 \mathrm{~S} . \mathrm{m}^{-1}$ for the $\mathrm{CB} / \mathrm{PVdF}$ phase. These values are remarkably close to the experimental conductivity value $\left(250 \mathrm{~S} . \mathrm{m}^{-1}\right)$ measured in [55] on macroscopic PVdF/CB film exhibiting the same weight ratio as those in the electrodes studied here. The ratios measured in [55] are all displayed in the $\mathrm{x}$ axis of Figure 6a. The ratio that was used on the electrodes 
(F, $\mathrm{H}$ and $\mathrm{J}$ ) is 5:3 (in bold characters in Figure 6a). This suggests that this PVdF/CB mixture shows similar electrical properties when forming a bulky macroscopic film or when it is distributed as micrometric clusters within a composite electrode (as in our study). This apparent very good agreement between experimental and calculated PVdF/CB conductivity values could indicate (even though our subvolumes are not representative of the large NMC clusters) that the spatial distribution of the $\mathrm{CB} / \mathrm{PVdF}$ is representative. It may also imply that the spatial distribution of the large NMC clusters does not impact the effective conductivity enough to be fully taken into consideration. That may be why we do not need to capture the NMC cluster RVE (as discussed in section 5.3) to obtain relevant results as those we are presenting here.

Figure $6 \mathrm{~b}$ displays the cumulative volume fraction of the $\mathrm{CB} / \mathrm{PVdF}$ phase $v$ s. the local current density field in this phase for $\mathrm{F}$ and $\mathrm{H}$. This local current density field is extracted from the FFT computation results. It can be seen that the current is distributed over two decades as it varies typically between 5 and $100 \mathrm{~A} \cdot \mathrm{m}^{-2}$ [instead of $100 \mathrm{~A} \cdot \mathrm{m}^{-1}$ ] when the cumulative volume fraction increases from about 10 to $80 \%$. In sub-volume $\mathrm{F}$, a less intense current streams through the $\mathrm{CB} / \mathrm{PVdF}$ phase compared to sub-volume $\mathrm{H}$. This is consistent with the two sub-volumes having the same tortuosity but more $\mathrm{PVdF} / \mathrm{CB}$ in $\mathrm{F}$ than in $\mathrm{H}$, with also a slightly higher intraconnectivity for this phase in sub-volume F.

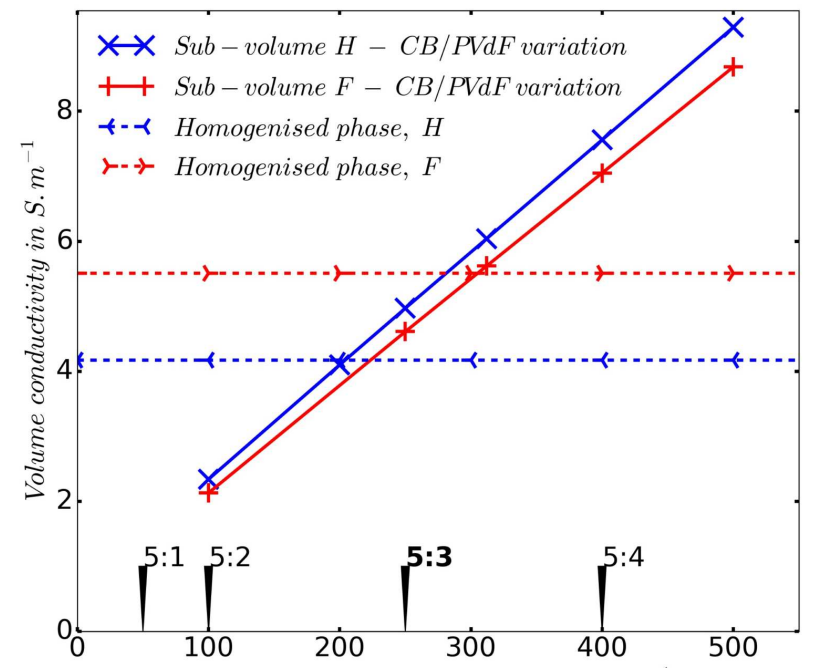

a)

Figure 6: a) Computed volume conductivity for the sub-volumes from samples $\mathrm{F}$ and $\mathrm{H}$ as a function of the PVdF/CB phase conductivity. Dashed horizontal lines are the homogenised phase conductivity deduced from the previous simulations on the XRCT volumes. Vertical lines are experimental measurements for the $\mathrm{PVdF} / \mathrm{CB}$ phase from [55], ratios are $\mathrm{PVdF} / \mathrm{CB}$ weight ratios. The ratio used in our electrodes $\mathrm{F}, \mathrm{H}$ and $\mathrm{J}$ is the one written in bold font $(5: 3)$. b) Cumulative volume fraction of the $\mathrm{PVdF} / \mathrm{CB}$ phase as a function of the current value in this phase. Only the results for the lowest current density values are displayed.

Table 4: Simulation results and input/target parameters for the FFT simulations on FIB/SEM sub volumes. 


\begin{tabular}{|c|c|c|c|c|c|c|}
\hline \multirow[t]{2}{*}{ Sample } & \multicolumn{2}{|c|}{$\begin{array}{l}\text { Target: LFP, PVdF/CB } \\
\text { and porosity homogenised } \\
\text { phase conductivity } \\
\left({\left.\mathrm{S} . \mathrm{m}^{-1}\right)}^{-1}\right.\end{array}$} & \multicolumn{2}{|c|}{ Input } & \multicolumn{2}{|c|}{ Result } \\
\hline & $\begin{array}{c}\text { Experimentally } \\
\text { measured }\end{array}$ & $\begin{array}{c}\text { calculated } \\
\text { with XRCT } \\
\text { volume }\end{array}$ & $\begin{array}{c}\text { PVdF/CB } \\
\text { phase } \\
\text { conductivity } \\
\text { experimen- } \\
\text { tally } \\
\text { measured } \\
\left({\left.\mathrm{S} . \mathrm{m}^{-1}\right)}^{-1}\right.\end{array}$ & 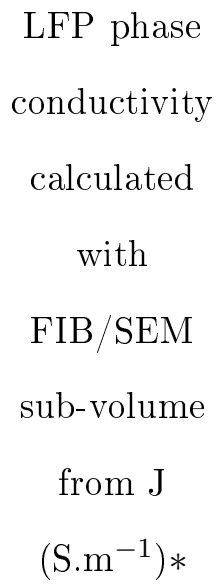 & $\begin{array}{c}\text { LFP phase } \\
\text { conductivity } \\
\left({\left.\mathrm{S} . \mathrm{m}^{-1}\right)}^{-1}\right.\end{array}$ & $\begin{array}{c}\mathrm{PVdF} / \mathrm{CB} \\
\text { phase } \\
\text { conductivity } \\
\left(\mathrm{S}^{-1} \mathrm{~m}^{-1}\right)\end{array}$ \\
\hline F & - & 5.51 & - & 0.38 & - & 305 \\
\hline $\mathrm{H}$ & - & 4.26 & - & 0.38 & - & 210 \\
\hline $\mathrm{F}$ & 0.3 & - & 250 & - & 0.38 & - \\
\hline
\end{tabular}

* Note that the LFP phase conductivity calculated with FIB/SEM sub-volume from J is very close to that experimentally measured on a LFP pellet (see in Table 2 the "carbon coating" conductivity value, which gives the effective conductivity of the carbon coated LFP material).

Finally, 2D maps of the current field, visualised for one slice of volumes $\mathrm{F}$ and $\mathbf{J}$ (at scale 2), are shown in Figure 7. A colour map is used to distinguish areas in which the application of a potential difference causes an enhanced electric current (in yellow and white) from areas where it is moderate or inexistent (in blue and black). The colour scale is different for $\mathrm{F}$, from 0 to $126{\mathrm{~A} . \mathrm{m}^{-2}}^{-2}$ instead of $126 \mathrm{~A} \cdot \mathrm{m}^{-1}$ ], than for J, from 0 to $6 \mathrm{~A} . \mathrm{m}^{-2}$. It is clearly visible in these images that the current mainly streams through the PVdF/CB phase when it is percolated ( $c f$. Sample F). This also clearly illustrates that the conductivity of the homogenised phase considered in the previous subsection is dominated by the percolated PVdF/CB mixture. In electrode $\mathrm{J}$, it is also clear that the current is more intense in the $\mathrm{PVdF} / \mathrm{CB}$ clusters even if it is not percolating in this case. 


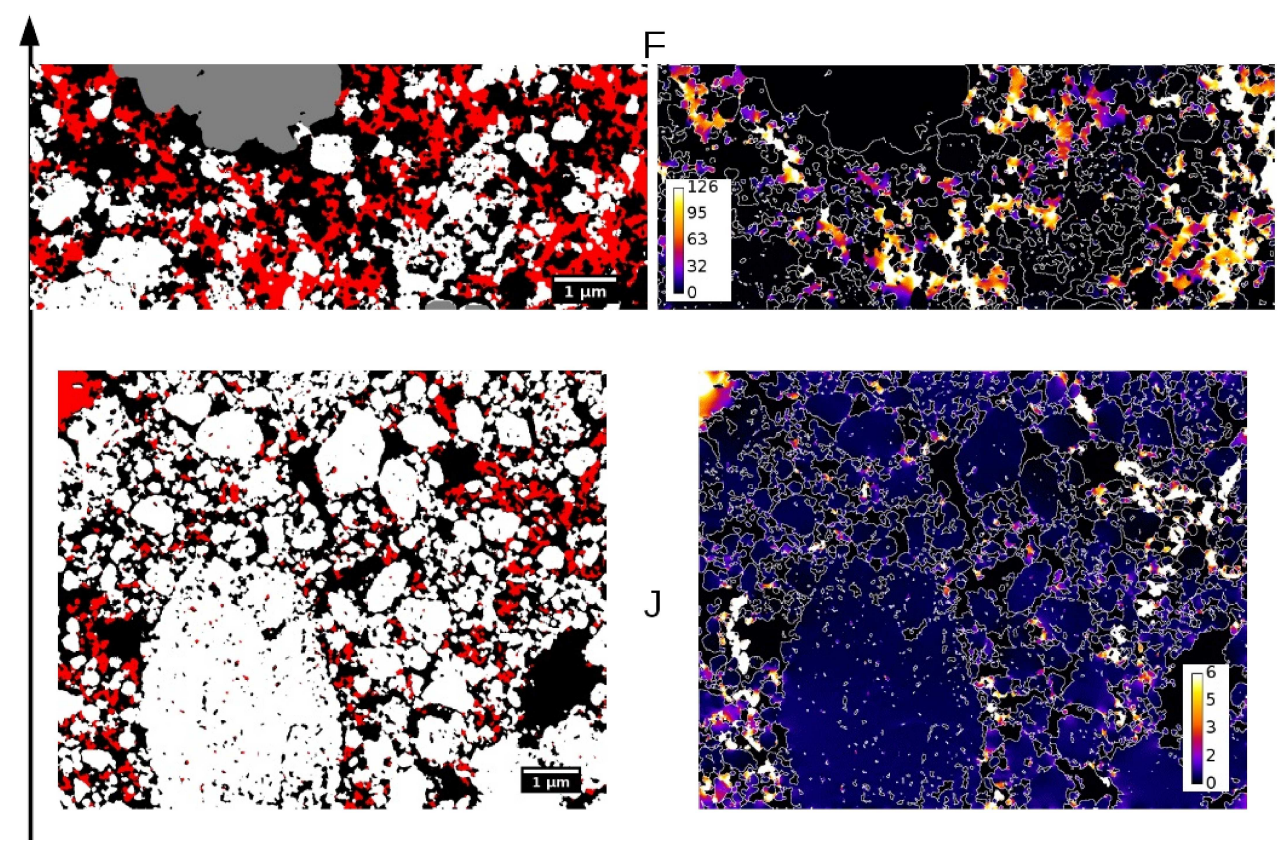

Figure 7: Segmented slice (left column) and 2D current maps (right column, FFT calculations) from one slice of the whole volumes, from top to bottom: Sample F and J. Colour scales for the current density are in A.m ${ }^{-2}$ [instead of A.m ${ }^{-1}$ ]. The left arrow indicates the direction of the imposed potential $(1 \mathrm{~V} / \mathrm{m})[$ instead of $1 \mathrm{~V}]$.

\subsection{Impact of the volume size on representativity}

Given its microstructure, the positive electrode can be seen as a random medium from a mathematical point of view. It means the heterogeneous electrode microstructure can be represented with an homogeneous medium characterised by a set of effective (i.e. macroscopic) parameters. Thus, the question of the representativeness of the analysed volumes is relevant, especially for the FIB/SEM volumes and sub-volumes. The simulated volumes must be large enough to characterise properly the effective behaviour of the global electrode. This is verified according to two different criteria in the following sections.

\subsubsection{Empirical criterion}

The more intuitive way to address this question is an assessment on the geometrical dimensions involved. As a rule of thumb (see for example [59, 31]), the volume is considered to be representative if its size is five to ten times larger than the so called "Elements of Interest" (EI) in the microstructure. Here, the EI are the NMC clusters at scale 1 and the LFP particles at scale 3. This size (mean diameter) has been measured on the three studied electrodes by image analysis using a local thickness algorithm within the Fiji software [60]. This rule of thumb statement tends to be nuanced by other considerations such as the size distribution heterogeneity as stated in [61]. Results are gathered in Table 5. The ratio between the lateral size of the EI 
and the lateral size of the analysed volume is also calculated in this table. In the three studied cases, this ratio is nearly equal or higher than 15 . These results might provide a first look at the representativeness of its simulation volumes. However, it is hard to firmly conclude on such a criterion if there is not a lot of volumes from the same source to converge on a statistical evaluation. This is why another criterion is developed in the next subsection in order to be more systematic and selective when only one volume, or a few, is available.

One can notice that, in Table 5, we do not take the thickness (the minimal dimension) of the XRCT volumes as the lateral size. This is because we have captured the entire electrode thickness in the XRCT volumes. This dimension is thus inherently exact and representative. Only the two other dimensions can possibly be non-representative. However, the dimension that is of primary importance in our simulation on XRCT volumes is the thickness, making all other analysis of their representativeness of little interest. That is why the mathematical evaluation discussed in the next paragraph (5.3.2) only concerns the FIB/SEM volumes and scale 3 .

Table 5: Geometrical analysis of the representativity of the simulation volumes. Comparison between the lateral size of the simulation volume and the "Element of Interest" (EI) in the microstructure, depending on the scale.

\begin{tabular}{c|c|c|c|c|c|c}
\multirow{2}{*}{ Sample } & \multicolumn{2}{|c|}{$\begin{array}{c}\text { Lateral size of the simulation } \\
\text { volume }(\mu \mathrm{m})\end{array}$} & \multicolumn{2}{c|}{$\begin{array}{c}\text { Characteristic length of the } \\
\text { EI }(\mu \mathrm{m})\end{array}$} & \multicolumn{2}{|c}{ Ratio } \\
\cline { 2 - 7 } & Scale 1 & Scale 2 & Scale 1 & Scale 2 & Scale 1 & Scale 2 \\
\hline $\mathrm{F}$ & 128 & 2.3 & 8.29 & 0.16 & 15.44 & 14.4 \\
\hline $\mathrm{H}$ & 128 & 4 & 7.27 & 0.15 & 17.61 & 26.7 \\
\hline $\mathrm{J}$ & 128 & 5 & - & 0.22 & - & 22.7
\end{tabular}

\subsubsection{Mathematical evaluation}

It is possible to evaluate the representativity of a volume by appealing to the theory originally developed by Matheron [62] and Lantuéjoul [63]. In this theory, "representativity" is estimated by examining how the variance of the mean of a spatial field over a volume decays with the volume size, as the later becomes very large. In the present context, the field is the local current and the mean is the apparent conductivity. Matheron's main result is that the variance decays proportionally to the inverse of $\mathrm{V}$, where $\mathrm{V}$ is the volume size, except in particular cases where the field presents correlations at infinite length scales. Matheron's theory, however, 
assumes that the field is stationary (statistically independent of translations). In all rigour, this condition is not fulfilled here, because of the effect of boundary conditions. Although Matheron's theorems do not rigorously apply to the present problem, this theory nevertheless provides a useful criterion for quantifying the representativity (see e.g. [64]).

Hereafter, we use sample $\mathrm{J}$ at scale 2 because its microstructure is similar to the one of the other samples and because it is the largest volume available containing only LFP, CB/PVdF and porosity. The whole simulation volume is divided in height equal parts, themselves divided in height equal parts and so on over several levels. Then, we compute the effective conductivity for each volume by using the same phase conductivities as in section $5.1\left(\mathrm{LFP}=0.38 \mathrm{~S} \cdot \mathrm{m}^{-1}, \mathrm{PVdF} / \mathrm{CB}=250 \mathrm{~S} \cdot \mathrm{m}^{-1}\right)$. The result of these calculations is shown in Figure 8). The variance $\mathrm{D}_{\sigma}^{2}(\mathrm{~V})$ is then calculated for each size level. According to [65, 62], to obtain a relative error $\varepsilon_{r e l}$ on the estimate of the effective volume conductivity, the computation volume $\mathrm{V}$ must be larger than about:

$$
V=\frac{4 D_{\sigma}^{2} A_{3}}{Z^{2} \varepsilon_{r e l}^{2}}
$$

with $\mathrm{Z}$, the effective conductivity value for the whole volume. $\mathrm{D}_{\sigma}^{2}$ is the point variance calculated across the whole volume, i.e. the variance of the local field $\sigma . \mathrm{A}_{3}$ is the integral range, a constant linked to the covariance function of the field [65]. In practice, $\mathrm{A}_{3}$ is determined by a fit of the curve $\mathrm{D}_{\sigma}^{2}(\mathrm{~V}) / \mathrm{D}_{\sigma}^{2}$ vs. $1 / \mathrm{V}$ (see Figure $8 \mathrm{~b}$ ). We obtain $\mathrm{A}_{3} \sim 2.46 \times 10^{5}$ (in voxels) for the sample studied here. 


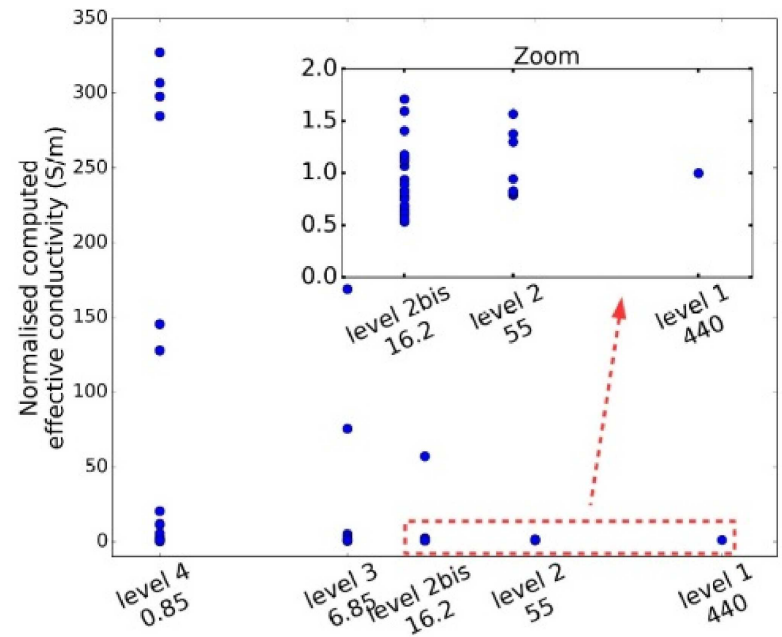

a)

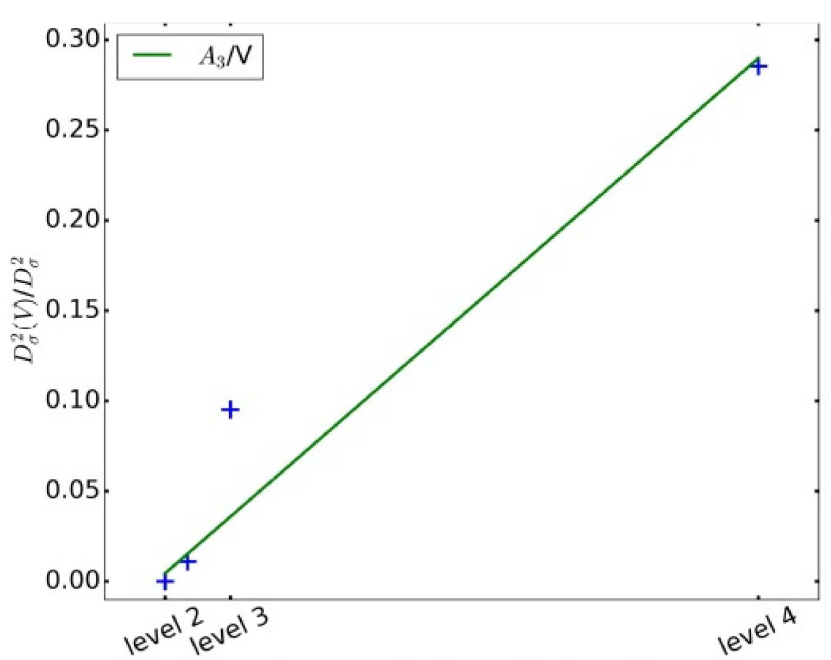

b)

Inverse of volume size in $\mu \mathrm{m}^{3}$

Figure 8: a) Evolution of the normalised computed effective conductivity (reference value is the computed effective conductivity for level 1) with the volume size. b) Evolution of the variance with the inverse of the volume size. Level 1 corresponds to electrode $\mathrm{J}$ at scale 2 .

According to FFT numerical results, the size of the RVE is $100 \times 100 \times 100 \mu \mathrm{m}^{3}$ when $\varepsilon_{\text {rel }}$ equals $10 \%$. Hence, the simulation volumes used in Section 5.2 are too small to be representative according to this statistical criterion with a relative error superior to $120 \%$. Equation 3 also allows us to calculate that for a volume similar in size to the one of electrode $\mathrm{J}$ (scale 2), we should use 15 different volumes in order to statistically extract the computed effective conductivity value for a relative tolerated error $\varepsilon_{\text {rel }}$ of $10 \%$ and 4 for a $\varepsilon_{r e l}$ of $20 \%$. Although not fully satisfactory from a statistical point of view, we however managed to compute realistic properties when comparing to experimental ones (see Table 2). We can also point out that the volume fractions of the different phases in the numerical volumes are close to experimental ones especially for samples $\mathrm{J}$ and $\mathrm{F}$. Although our volume is smaller than the theoretical statistical RVE, the geometrical criterion is not totally conclusive but the results match the experimental measurements. This may be the result of the $\mathrm{CB} / \mathrm{PVdF}$ spatial distribution at the FIB/SEM scale being representative of the one at the other scales. Clearly, we would need several FIB-SEM imaging to assess representativity.

\subsection{Analytical estimates}

In this part, we use the results of our simulations, more particularly the conductivity values of the different phases, to evaluate classical analytical approaches for calculating the effective conductivity of composite materials. Indeed, it is not always possible to make use of multiple experimental techniques as sophisticated as XR and FIB/SEM tomography, and to couple them with numerical simulations, to estimate the effective conductivity of a composite material from the values of its different phases. Simple analytical approaches 
can therefore be useful for the engineer.

Among all the literature, we first consider the Voigt and Reuss bounds, which are parallel and series resistance circuit analogy respectively. We have then the following equations 4 and 5 , where $\sigma_{v o l}$ is the conductivity of the composite volume and $\phi_{v}$ the phase volume fraction.

$$
\begin{gathered}
\sigma_{v o l, \text { Voigt }}=\phi_{v, N M C} \sigma_{N M C}+\phi_{v, h o m o g} \sigma_{h o m o g} \\
\sigma_{v o l, \text { Reuss }}=\frac{\sigma_{N M C} \sigma_{\text {homog }}}{\phi_{v, N M C} \sigma_{\text {homog }}+\phi_{v, h o m o g} \sigma_{N M C}}
\end{gathered}
$$

More narrow bounds are obtained for isotropic two-phase media, which lead to equations 6 and 7 [66].

$$
\begin{gathered}
\sigma_{v o l, H \text { Smin }}=\sigma_{N M C}\left(1+\frac{\phi_{v, h o m o g}}{\frac{\sigma_{N M C}}{\sigma_{\text {homog }}-\sigma_{N M C}}+\frac{\phi_{v, N M C}}{3}}\right) \\
\sigma_{v o l, H \text { Smax }}=\sigma_{\text {homog }}\left(1+\frac{\phi_{v, N M C}}{\frac{\sigma_{\text {homog }}}{\sigma_{N M C}-\sigma_{\text {homog }}}+\frac{\phi_{v, h o m o g}}{3}}\right)
\end{gathered}
$$

We also make use of the Self Consistent (SC) approximation [67]. In the limiting case where the NMC conductivity vanishes, it gives equation 8 .

$$
\sigma_{S C}=\left(1-\phi_{v, N M C}-\frac{\phi_{v, N M C}}{2}\right) \sigma_{h o m o g}
$$

We consider here scale 1 where the volumes are modelled as a mixture of the NMC phase and a second homogeneous one containing, at a lower scale, the LFP, PVdF/CB and porosity phases. We use 0.0001S.m ${ }^{-1}$ as the phase conductivity for NMC ( $\sigma_{N M C}, c f$. Table 2). As in Section 5.1, we let the conductivity of the homogenised phase vary and determine the value of $\sigma_{\text {homog }}$ so that analytical predictions match experimentally-measured values. We focus here on sample F, as an example. Results for the analytical simulations are plotted in Figure 9 along with the experimental value to fit. Results are similar to the FFT ones for the upper bounds (Voigt and Hashin-Shtrikman) and the SC estimate. As expected, the lower bounds (Reuss and Hashin-Shtrikman) are close to zero. The numerical values calculated using a fit of the experimental values and that of the FTT predictions on the same sample are compared in Table 6. We observe excellent agreement between SC estimates and FFT predictions. 


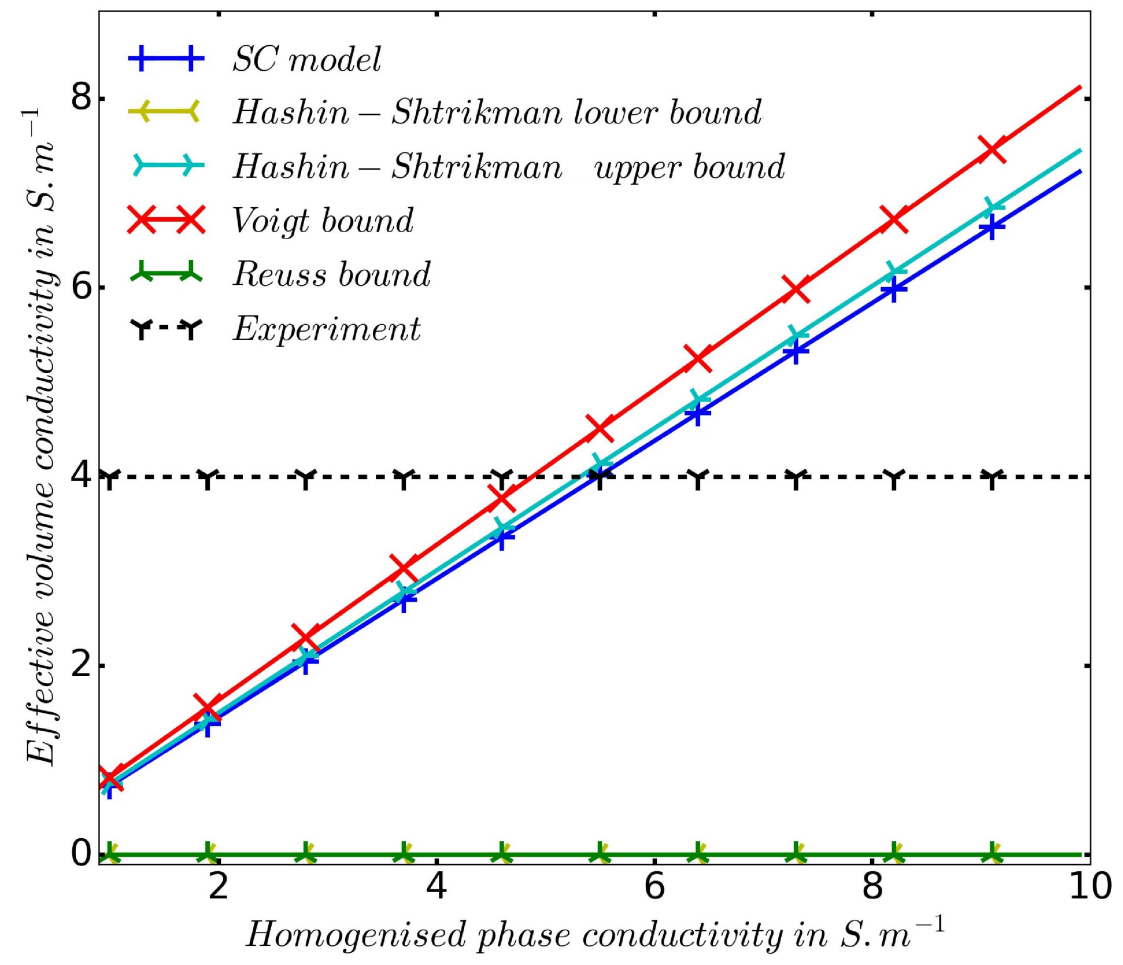

Figure 9: Variation of the analytical models with the conductivity of the homogenised phase for XRCT sample $\mathrm{F}$ along with the measured experimental global conductivity value

Table 6: Analytical and FFT simulation results for the homogenised phase conductivity in order to fit the experimentally measured effective conductivity of sample F.

\begin{tabular}{|c|c|c|c|c|c|c|}
\hline Sample & $\begin{array}{c}\text { Voigt } \\
\left(\text { S.m }{ }^{-1}\right)\end{array}$ & $\begin{array}{c}\text { Hashin- } \\
\text { Shtrikman } \\
\text { upper } \\
\left({\left.\mathrm{S} . \mathrm{m}^{-1}\right)}^{-1}\right.\end{array}$ & $\begin{array}{c}\text { Reuss } \\
\left(\mathrm{S} . \mathrm{m}^{-1}\right)\end{array}$ & $\begin{array}{c}\text { Hashin- } \\
\text { Shtrikman } \\
\text { lower } \\
\left({\left.\mathrm{S} . \mathrm{m}^{-1}\right)}^{-1}\right.\end{array}$ & $\begin{array}{c}\text { SC model } \\
\left({\left.\mathrm{S} . \mathrm{m}^{-1}\right)}^{-1}\right.\end{array}$ & 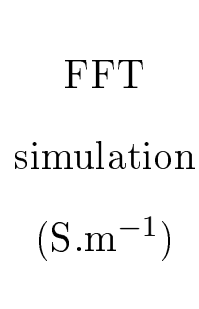 \\
\hline $\mathrm{F}$ & 4.88 & 5.32 & $2.53 \times 10^{8}$ & $2.81 \times 10^{7}$ & 5.48 & 5.51 \\
\hline
\end{tabular}

At the large field of view containing the NMC clusters (noted scale 1), the microstructure can be assumed as nearly non conducting spherical particles embedded in a conducting matrix. In this case, the upper bounds and the SC estimate lead to good predictions as we remain close to the hypothesis on which they are based. If we take into consideration the real microstructure at the finer scales (scale 2 and 3 ) this is no longer the case. As examples, let us consider electrode $\mathrm{J}$ and $\mathrm{F}$, at scale 3 , in order to compare cases where there is 
percolation $(\mathrm{F})$ or not $(\mathrm{J})$. At these scales, the electrodes are composed by three phases, the LFP active material, the PVdF/CB mixture and the porosity. Only the Voigt and Reuss bounds and the SC estimate can be extended to a three phase material, the Hashin and Shtrikman bounds are thus not calculated in the following. From these analytical tools, we try to predict the effective volume conductivity based on the phase properties we determined earlier (i.e. $0.38 \mathrm{~S} . \mathrm{m}^{-1}$ for the LFP, $250 \mathrm{~S} . \mathrm{m}^{-1}$ for the PVdF $/ \mathrm{CB}$ mixture and $0 \mathrm{~S} . \mathrm{m}^{-1}$ for the porosity). Results are gathered in Table 7 with the FFT predictions and experimental values to be compared with. As one can see, results show not so good agreement for electrode J and really bad agreement for electrode F. This can simply be explained by the fact that real microstructures no longer fit, at these finer scales, into the assumptions that underlie analytical models.

Table 7: Analytical and FFT prediction results or experimental measurements for the effective volume conductivity at scale 3 for electrodes $\mathrm{J}$ and $\mathrm{F}$.

\begin{tabular}{c|c|c|c|c|c} 
Sample & $\begin{array}{c}\text { Voigt } \\
\left(\mathrm{S} . \mathrm{m}^{-1}\right)\end{array}$ & $\begin{array}{c}\text { Reuss } \\
\left(\mathrm{S} . \mathrm{m}^{-1}\right)\end{array}$ & $\begin{array}{c}\mathrm{SC} \text { model } \\
\left(\mathrm{S} . \mathrm{m}^{-1}\right)\end{array}$ & $\begin{array}{c}\mathrm{FFT} \\
\text { simulation } \\
\left(\mathrm{S} . \mathrm{m}^{-1}\right)\end{array}$ & $\begin{array}{c}\text { Experimental } \\
\text { measurements } \\
\left({\left.\mathrm{S} . \mathrm{m}^{-1}\right)}^{-1}\right.\end{array}$ \\
\hline $\mathrm{F}$ & 46.4 & 0 & 0.29 & 5.51 & - \\
\hline $\mathrm{J}$ & 12 & 0 & 0.24 & 0.3 & 0.3
\end{tabular}

\section{Conclusion}

In this work, we have used an FFT based numerical method coupled with experimental measurements, to characterise the influence of an electrode microstructure on its electronic conduction properties. As shown here, one may predict the electronic conductivity of battery composite electrodes through FFT simulations by using a combination of X-Ray and FIB/SEM segmented volumes. The accuracy of this technique depends on the different scales in the microstructure. Based on the experimental measurement of an electrode conductivity and its segmented X-Ray and FIB/SEM volume, it was possible to back-calculate the conductivity of the PVdF/CB mixture and the LFP phase. Considering the variability of the microstructure, the volumes analysed using FIB/SEM are not fully representative but the values obtained are in good agreement with the experimental measurements made on these materials taken separately. This suggests the $\mathrm{CB} / \mathrm{PVdF}$ spatial distribution at the FIB/SEM small scale is actually representative of the spatial distribution of this conducting phase at the more global scales (like X-Ray tomography). The opposite approach is also possible, namely to predict the conductivity of an electrode starting from its segmented FIB/SEM volume and experimental 
measurements of the conductivities of the various materials that compose it.

This method allows one to predict local current paths in the microstructure. It is however crucial to address the representativity of the microstructure as the numerical results are based on it. In this regard, tests to characterise the representativeness of the volumes were presented. According to our statistical analysis, the FIB/SEM simulation volumes used are too small to be fully representative. Nevertheless, even with a rough precision, FFT predictions are in good agreement with experimental values.

Analytical bounds and the classic self-consistent estimate also give accurate results for the effective properties at the XRCT scale. These tools may then be of some use to monitor the accuracy of numerical simulations or to simply access or assess some values in a multi-scale approach. However, at finer scales and for more complexe microstructures, these methods have shown their limitations.

This study opens several new perspectives, such as the calculation of the effective conductivity of an electrode from its real microstructure and the conductivity of its different phases, in order to better understand the effect of the electrode formulation, or of various mechanical or physico-chemical stimuli applied to the electrode during its manufacture or battery operation.

\section{Acknowledgement}

We are grateful to the ANR for the funding of the Pepite project (ANR-15-CE05-0001).

\section{References}

[1] D. Andre, S.-J. Kim, P. Lamp, S. F. Lux, F. Maglia, O. Paschos, and B. Stiaszny, "Future generations of cathode materials: an automotive industry perspective," Journal of Materials Chemistry A, vol. 3, no. 13, pp. 6709-6732, 2015.

[2] J. W. Choi and D. Aurbach, "Promise and reality of post-lithium-ion batteries with high energy densities," Nature Reviews Materials, vol. 1, Apr. 2016.

[3] N. Besnard, A. Etiemble, T. Douillard, O. Dubrunfaut, P. Tran-Van, L. Gautier, S. Franger, J.-C. Badot, E. Maire, and B. Lestriez, "Multiscale Morphological and Electrical Characterization of Charge Transport Limitations to the Power Performance of Positive Electrode Blends for Lithium-Ion Batteries," Advanced Energy Materials, vol. 7, no. 8, 2017. 
[4] M. Katayama, K. Sumiwaka, R. Miyahara, H. Yamashige, H. Arai, Y. Uchimoto, T. Ohta, Y. Inada, and Z. Ogumi, "X-ray absorption fine structure imaging of inhomogeneous electrode reaction in $\mathrm{LiFePO}_{4}$ lithium-ion battery cathode," Journal of Power Sources, vol. 269, pp. 994-999, Dec. 2014.

[5] H. Liu, J. M. Foster, A. Gully, S. Krachkovskiy, M. Jiang, Y. Wu, X. Yang, B. Protas, G. R. Goward, and G. A. Botton, "Three-dimensional investigation of cycling-induced microstructural changes in lithiumion battery cathodes using focused ion beam/scanning electron microscopy," Journal of Power Sources, vol. 306, pp. 300-308, Feb. 2016.

[6] S. L. Morelly, N. J. Alvarez, and M. H. Tang, "Short-range contacts govern the performance of industryrelevant battery cathodes," Journal of Power Sources, vol. 387, pp. 49-56, May 2018.

[7] D. Robert, T. Douillard, A. Boulineau, G. Brunetti, P. Nowakowski, D. Venet, P. Bayle-Guillemaud, and C. Cayron, "Multiscale Phase Mapping of $\mathrm{LiFePO}_{4}$-Based Electrodes by Transmission Electron Microscopy and Electron Forward Scattering Diffraction," ACS Nano, vol. 7, pp. 10887-10894, Dec. 2013.

[8] M. R. Roberts, A. Madsen, C. Nicklin, J. Rawle, M. G. Palmer, J. R. Owen, and A. L. Hector, "Direct Observation of Active Material Concentration Gradients and Crystallinity Breakdown in $\mathrm{LiFePO}_{4}$ Electrodes During Charge/Discharge Cycling of Lithium Batteries," The Journal of Physical Chemistry C, vol. 118, pp. 6548-6557, Apr. 2014.

[9] Y.-c. K. Chen-Wiegart, R. DeMike, C. Erdonmez, K. Thornton, S. A. Barnett, and J. Wang, "Tortuosity characterization of 3d microstructure at nano-scale for energy storage and conversion materials," Journal of Power Sources, vol. 249, pp. 349-356, Mar. 2014.

[10] M. Ebner, F. Geldmacher, F. Marone, M. Stampanoni, and V. Wood, "X-Ray Tomography of Porous, Transition Metal Oxide Based Lithium Ion Battery Electrodes," Advanced Energy Materials, vol. 3, pp. 845-850, July 2013.

[11] M. Ender, J. Joos, A. Weber, and E. Ivers-Tiffée, "Anode microstructures from high-energy and highpower lithium-ion cylindrical cells obtained by X-ray nano-tomography," Journal of Power Sources, vol. 269, pp. 912-919, Dec. 2014.

[12] A. Etiemble, N. Besnard, A. Bonnin, J. Adrien, T. Douillard, P. Tran-Van, L. Gautier, J.-C. Badot, E. Maire, and B. Lestriez, "Multiscale morphological characterization of process induced heterogeneities in blended positive electrodes for lithium-ion batteries," Journal of Materials Science, vol. 52, pp. 35763596, Apr. 2017. 
[13] T. Hutzenlaub, S. Thiele, R. Zengerle, and C. Ziegler, "Three-Dimensional Reconstruction of a $\mathrm{LiCoO}_{2}$ Li-Ion Battery Cathode," Electrochemical and Solid-State Letters, vol. 15, no. 3, p. A33, 2012.

[14] Z. Liu, J. Scott Cronin, Y.-c. K. Chen-Wiegart, J. R. Wilson, K. J. Yakal-Kremski, J. Wang, K. T. Faber, and S. A. Barnett, "Three-dimensional morphological measurements of $\mathrm{LiCoO}_{2}$ and $\mathrm{LiCoO}_{2} / \mathrm{Li}\left(\mathrm{Ni}_{1 / 3} \mathrm{Mn}_{1 / 3} \mathrm{Co}_{1 / 3}\right) \mathrm{O}_{2}$ lithium-ion battery cathodes," Journal of Power Sources, vol. 227, pp. 267-274, Apr. 2013.

[15] P. Shearing, L. Howard, P. Jørgensen, N. Brandon, and S. Harris, "Characterization of the 3-dimensional microstructure of a graphite negative electrode from a Li-ion battery," Electrochemistry Communications, vol. 12, pp. 374-377, Mar. 2010.

[16] J. R. Wilson, J. S. Cronin, S. A. Barnett, and S. J. Harris, "Measurement of three-dimensional microstructure in a $\mathrm{LiCoO}_{2}$ positive electrode," Journal of Power Sources, vol. 196, pp. 3443-3447, Apr. 2011.

[17] L. Zielke, T. Hutzenlaub, D. R. Wheeler, I. Manke, T. Arlt, N. Paust, R. Zengerle, and S. Thiele, "A Combination of X-Ray Tomography and Carbon Binder Modeling: Reconstructing the Three Phases of $\mathrm{LiCoO}_{2}$ Li-Ion Battery Cathodes," Advanced Energy Materials, vol. 4, p. 1301617, June 2014.

[18] S. Cooper, D. Eastwood, J. Gelb, G. Damblanc, D. Brett, R. Bradley, P. Withers, P. Lee, A. Marquis, N. Brandon, and P. Shearing, "Image based modelling of microstructural heterogeneity in $\mathrm{LiFePO}_{4}$ electrodes for Li-ion batteries," Journal of Power Sources, vol. 247, pp. 1033-1039, Feb. 2014.

[19] T. Hutzenlaub, S. Thiele, N. Paust, R. Spotnitz, R. Zengerle, and C. Walchshofer, "Three-dimensional electrochemical Li-ion battery modelling featuring a focused ion-beam/scanning electron microscopy based three-phase reconstruction of a $\mathrm{LiCoO}_{2}$ cathode," Electrochimica Acta, vol. 115, pp. 131-139, Jan. 2014.

[20] A. G. Kashkooli, S. Farhad, D. U. Lee, K. Feng, S. Litster, S. K. Babu, L. Zhu, and Z. Chen, "Multiscale modeling of lithium-ion battery electrodes based on nano-scale X-ray computed tomography," Journal of Power Sources, vol. 307, pp. 496-509, Mar. 2016.

[21] A. H. Wiedemann, G. M. Goldin, S. A. Barnett, H. Zhu, and R. J. Kee, "Effects of three-dimensional cathode microstructure on the performance of lithium-ion battery cathodes," Electrochimica Acta, vol. 88 , pp. 580-588, Jan. 2013. 
[22] B. Yan, C. Lim, L. Yin, and L. Zhu, "Three Dimensional Simulation of Galvanostatic Discharge of $\mathrm{LiCoO}_{2}$ Cathode Based on X-ray Nano-CT Images," Journal of the Electrochemical Society, vol. 159, pp. A1604-A1614, Aug. 2012.

[23] K. Higa, S.-L. Wu, D. Y. Parkinson, Y. Fu, S. Ferreira, V. Battaglia, and V. Srinivasan, "Comparing Macroscale and Microscale Simulations of Porous Battery Electrodes," Journal of the Electrochemical Society, vol. 164, pp. E3473-E3488, June 2017.

[24] R. Dominko, M. Gaberšček, J. Drofenik, M. Bele, and S. Pejovnik, "A Novel Coating Technology for Preparation of Cathodes in Li-Ion Batteries," Electrochemical and Solid-State Letters, vol. 4, no. 11, p. A187, 2001.

[25] A. M. Grillet, T. Humplik, E. K. Stirrup, S. A. Roberts, D. A. Barringer, C. M. Snyder, M. R. Janvrin, and C. A. Apblett, "Conductivity Degradation of Polyvinylidene Fluoride Composite Binder during Cycling: Measurements and Simulations for Lithium-Ion Batteries," J. Electrochem. Soc., vol. 163, pp. A1859-A1871, Jan. 2016.

[26] D. Guy, B. Lestriez, R. Bouchet, and D. Guyomard, "Critical role of polymeric binders on the electronic transport properties of composites electrode," Journal of The Electrochemical Society, vol. 153, no. 4, pp. A679-A688, 2006.

[27] M. G. Lazarraga, S. Mandal, J. Ibañez, J. Manuel Amarilla, and J. M. Rojo, "LiMn $2 \mathrm{O}_{4}$-based composites processed by a chemical-route Microstructural, electrical, electrochemical, and mechanical characterization," Journal of Power Sources, vol. 115, pp. 315-322, Apr. 2003.

[28] D. R. Nevers, S. W. Peterson, L. Robertson, C. Chubbuck, J. Flygare, K. Cole, and D. R. Wheeler, "The Effect of Carbon Additives on the Microstructure and Conductivity of Alkaline Battery Cathodes," Journal of the Electrochemical Society, vol. 161, pp. A1691-A1697, July 2014.

[29] R. Scipioni, P. S. Jørgensen, D.-T. Ngo, S. B. Simonsen, Z. Liu, K. J. Yakal-Kremski, H. Wang, J. Hjelm, P. Norby, S. A. Barnett, and S. H. Jensen, "Electron microscopy investigations of changes in morphology and conductivity of $\mathrm{LiFePO}_{4}$ /C electrodes," Journal of Power Sources, vol. 307, pp. 259-269, Mar. 2016.

[30] Y.-H. Chen, C.-W. Wang, G. Liu, X.-Y. Song, V. S. Battaglia, and A. M. Sastry, "Selection of Conductive Additives in Li-Ion Battery Cathodes," Journal of The Electrochemical Society, vol. 154, no. 10, p. A978, 2007. 
[31] A. Awarke, S. Lauer, M. Wittler, and S. Pischinger, "Quantifying the effects of strains on the conductivity and porosity of $\mathrm{LiFePO}_{4}$ based Li-ion composite cathodes using a multi-scale approach," Computational Materials Science, vol. 50, pp. 871-879, Jan. 2011.

[32] A. Awarke, S. Lauer, S. Pischinger, and M. Wittler, "Percolation-tunneling modeling for the study of the electric conductivity in $\mathrm{LiFePO}_{4}$ based Li-ion battery cathodes," Journal of Power Sources, vol. 196, pp. 405-411, Jan. 2011.

[33] W. Haselrieder, S. Ivanov, H. Tran, S. Theil, L. Froböse, B. Westphal, M. Wohlfahrt-Mehrens, and A. Kwade, "Influence of formulation method and related processes on structural, electrical and electrochemical properties of LMS/NCA-blend electrodes," Progress in Solid State Chemistry, vol. 42, pp. 157174, Dec. 2014.

[34] F. L. E. Usseglio-Viretta, A. Colclasure, A. N. Mistry, K. P. Y. Claver, F. Pouraghajan, D. P. Finegan, T. M. M. Heenan, D. Abraham, P. Mukherjee, D. Wheeler, P. Shearing, S. J. Cooper, K. Smith, "Resolving the Discrepancy in Tortuosity Factor Estimation for Li-Ion Battery Electrodes through MicroMacro Modeling and Experiment," Journal of The Electrochemical Society, vol. 165, pp. A3403-A3426, Nov. 2018.

[35] A. N. Mistry, K. Smith, P. Mukherjee, "Secondary-Phase Stochastics in Lithium-Ion Battery Electrodes," ACS Applied Materials 63 Interfaces, vol. 10, pp. 6317-6326, Feb. 2018.

[36] C. Wieser, T. Prill, and K. Schladitz, "Multiscale simulation process and application to additives in porous composite battery electrodes," Journal of Power Sources, vol. 277, pp. 64-75, Mar. 2015.

[37] G. Inoue and M. Kawase, "Numerical and experimental evaluation of the relationship between porous electrode structure and effective conductivity of ions and electrons in lithium-ion batteries," Journal of Power Sources, vol. 342, pp. 476-488, Feb. 2017.

[38] J. M. Foster, A. Gully, H. Liu, S. Krachkovskiy, Y. Wu, S. B. Schougaard, M. Jiang, G. Goward, G. A. Botton, and B. Protas, "Homogenization Study of the Effects of Cycling on the Electronic Conductivity of Commercial Lithium-Ion Battery Cathodes," The Journal of Physical Chemistry C, vol. 119, pp. 1219912208, June 2015.

[39] A. Gully, H. Liu, S. Srinivasan, A. K. Sethurajan, S. Schougaard, and B. Protas, "Effective Transport Properties of Porous Electrochemical Materials - A Homogenization Approach," Journal of The Electrochemical Society, vol. 161, no. 8, pp. E3066-E3077, 2014. 
[40] D. Masson, B. Abdallah, F. Willot, D. Jeulin, E. Mercadelli, A. Sanson, A. Chesnaud, and A. Thorel, "Morphological Modelling of a Metal Foam Supported SOFC Configuration," ECS Transactions, vol. 68, pp. 2951-2960, July 2015.

[41] H. Moulinec and P. Suquet, "A fast numerical method for computing the linear and nonlinear properties of composites," C. R. Acad. Sc. Paris, vol. 318, pp. 1417-1423., 1994.

[42] D. J. Eyre and G. W. Milton, "A fast numerical scheme for computing the response of composites using grid refinement," The European Physical Journal Applied Physics, vol. 6, pp. 41-47, Apr. 1999.

[43] F. Willot, B. Abdallah, and Y.-P. Pellegrini, "Fourier-based schemes with modified Green operator for computing the electrical response of heterogeneous media with accurate local fields," International Journal for Numerical Methods in Engineering, vol. 98, pp. 518-533, May 2014.

[44] T. Prill, K. Schladitz, D. Jeulin, M. Faessel, and C. Wieser, "Morphological segmentation of FIB-SEM data of highly porous media," Journal of Microscopy, vol. 250, pp. 77-87, May 2013.

[45] M. E. Spahr, D. Goers, A. Leone, S. Stallone, and E. Grivei, "Development of carbon conductive additives for advanced lithium ion batteries," Journal of Power Sources, vol. 196, pp. 3404-3413, Apr. 2011.

[46] M. Yoo, C. W. Frank, and S. Mori, "Interaction of Poly(vinylidene fluoride) with Graphite Particles. 1. Surface Morphology of a Composite Film and Its Relation to Processing Parameters," Chem. Mater., vol. 15, pp. 850-861, Feb. 2003.

[47] M. Yoo, C. W. Frank, S. Mori, and S. Yamaguchi, "Interaction of Poly(vinylidene fluoride) with Graphite Particles. 2. Effect of Solvent Evaporation Kinetics and Chemical Properties of PVDF on the Surface Morphology of a Composite Film and Its Relation to Electrochemical Performance," Chem. Mater., vol. 16, pp. 1945-1953, May 2004.

[48] G. Liu, H. Zheng, X. Song, and V. S. Battaglia, "Particles and Polymer Binder Interaction: A Controlling Factor in Lithium-Ion Electrode Performance," J. Electrochem. Soc., vol. 159, pp. A214-A221, Jan. 2012.

[49] Vierrath, Severin, L. Zielke, R. Moroni, A. Mondon, D. R. Wheeler, R. Zengerle, and S. Thiele, "Morphology of nanoporous carbon-binder domains in Li-ion batteries - A FIB-SEM study," Electrochemistry Communications, vol. 60, pp. 176-179, , Nov. 2015

[50] J.-C. Badot, B. Lestriez, and O. Dubrunfaut, "Interest in broadband dielectric spectroscopy to study the electronic transport in materials for lithium batteries," Materials Science and Engineering: B, vol. 213, pp. 190-198, Nov. 2016. 
[51] K. A. Seid, J.-C. Badot, O. Dubrunfaut, S. Levasseur, D. Guyomard, and B. Lestriez, "Multiscale electronic transport mechanism and true conductivities in amorphous carbon-LiFePO${ }_{4}$ nanocomposites," J. Mater. Chem., vol. 22, pp. 2641-2649, Jan. 2012.

[52] X. Wang, L. Zhi, and K. MÃ(Ellen, "Transparent, Conductive Graphene Electrodes for Dye-Sensitized Solar Cells," Nano Lett., vol. 8, pp. 323-327, Jan. 2008.

[53] K. A. Seid, J. C. Badot, O. Dubrunfaut, M. T. Caldes, N. Stephant, L. Gautier, D. Guyomard, and B. Lestriez, "Multiscale electronic transport in $\mathrm{Li}_{1+x} \mathrm{Ni}_{1 / 3-u} \mathrm{Co}_{1 / 3-v} \mathrm{Mn}_{1 / 3-w} \mathrm{O}_{2}$ : a broadband dielectric study from $40 \mathrm{~Hz}$ to $10 \mathrm{GHz}, "$ Phys. Chem. Chem. Phys., vol. 15, pp. 19790-19798, Oct. 2013.

[54] J.-C. Badot, É. Ligneel, O. Dubrunfaut, D. Guyomard, and B. Lestriez, "A Multiscale Description of the Electronic Transport within the Hierarchical Architecture of a Composite Electrode for Lithium Batteries," Advanced Functional Materials, vol. 19, no. 17, pp. 2749-2758, 2009.

[55] H. Zheng, R. Yang, G. Liu, X. Song, and V. S. Battaglia, "Cooperation between Active Material, Polymeric Binder and Conductive Carbon Additive in Lithium Ion Battery Cathode," The Journal of Physical Chemistry C, vol. 116, pp. 4875-4882, Feb. 2012.

[56] A. Ambos, F. Willot, D. Jeulin, and H. Trumel, "Numerical modeling of the thermal expansion of an energetic material," International Journal of Solids and Structures, vol. 60-61, pp. 125-139, May 2015.

[57] F. Willot, "Fourier-based schemes for computing the mechanical response of composites with accurate local fields," Comptes Rendus Mécanique, vol. 343, pp. 232-245, Mar. 2015.

[58] D. Azzimonti, F. Willot, and D. Jeulin, "Optical properties of deposit models for paints: full-fields FFT computations and representative volume element," Journal of Modern Optics, vol. 60, pp. 519-528, Apr. 2013.

[59] A. Awarke, M. Wittler, S. Pischinger, and J. Bockstette, "A 3d mesoscale model of the collector-electrode interface in Li-ion batteries," Journal of The Electrochemical Society, vol. 159, no. 6, pp. A798-A808, 2012.

[60] "Fiji is just ImageJ." URL: https://imagej.net/Fiji, accessed june 5, 2019.

[61] F. L.E. Usseglio-Viretta, and K. Smith, "Quantitative Microstructure Characterization of a NMC Electrode," ECS Transactions, vol. 77, pp. 1095-1118, 2017.

[62] G. Matheron, "Random sets and integral geometry," 1975.

[63] C. Lantuéjoul, "Ergodicity and integral range," Journal of Microscopy, vol. 161, pp. 387-403, Mar. 1991. 
[64] B. Abdallah, F. Willot, and D. Jeulin, "Stokes Flow Through a Boolean Model of Spheres: Representative Volume Element," Transport in Porous Media, vol. 109, pp. 711-726, Sept. 2015.

[65] T. Kanit, S. Forest, I. Galliet, V. Mounoury, and D. Jeulin, "Determination of the size of the representative volume element for random composites: statistical and numerical approach," International Journal of Solids and Structures, vol. 40, pp. 3647-3679, June 2003.

[66] Z. Hashin and S. Shtrikman, "A variational approach to the theory of the elastic behaviour of polycrystals," Journal of the Mechanics and Physics of Solids, vol. 10, pp. 343-352, Oct. 1962.

[67] D. A. G. Bruggeman, "Berechnung verschiedener physikalischer Konstanten von heterogenen Substanzen. I. Dielektrizitätskonstanten und Leitfähigkeiten der Mischkörper aus isotropen Substanzen," Annalen der Physik, vol. 416, no. 7, pp. 636-664, 1935. 